
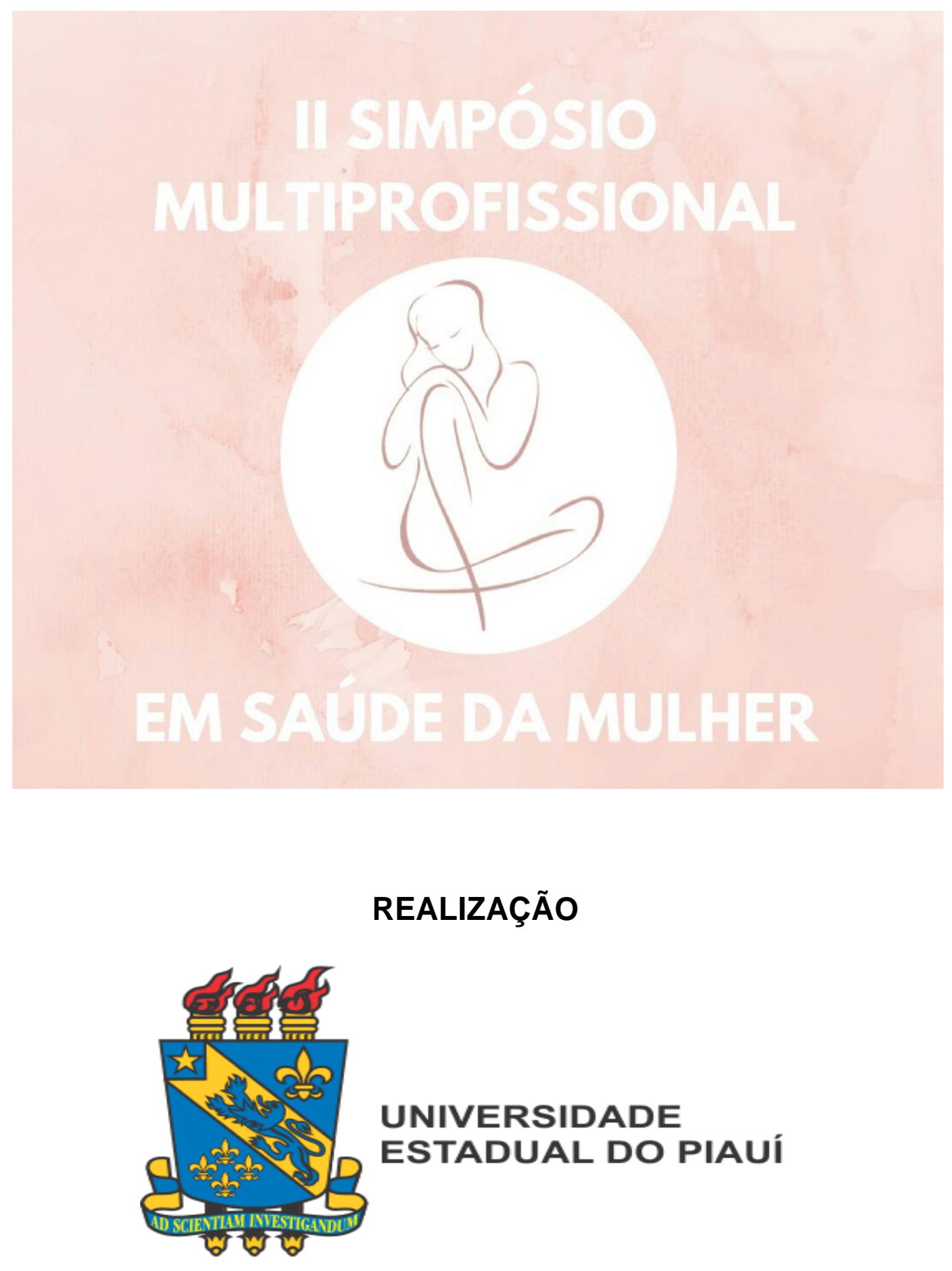

\title{
APOIO
}

\section{ACERVO mastiseristas}

As publicações mais rápidas do país!

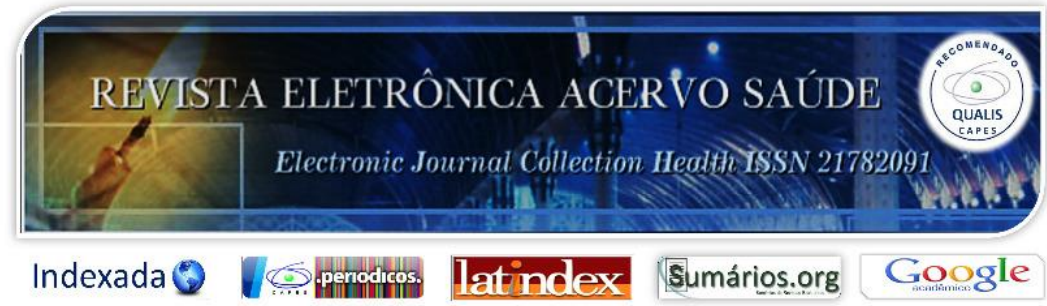




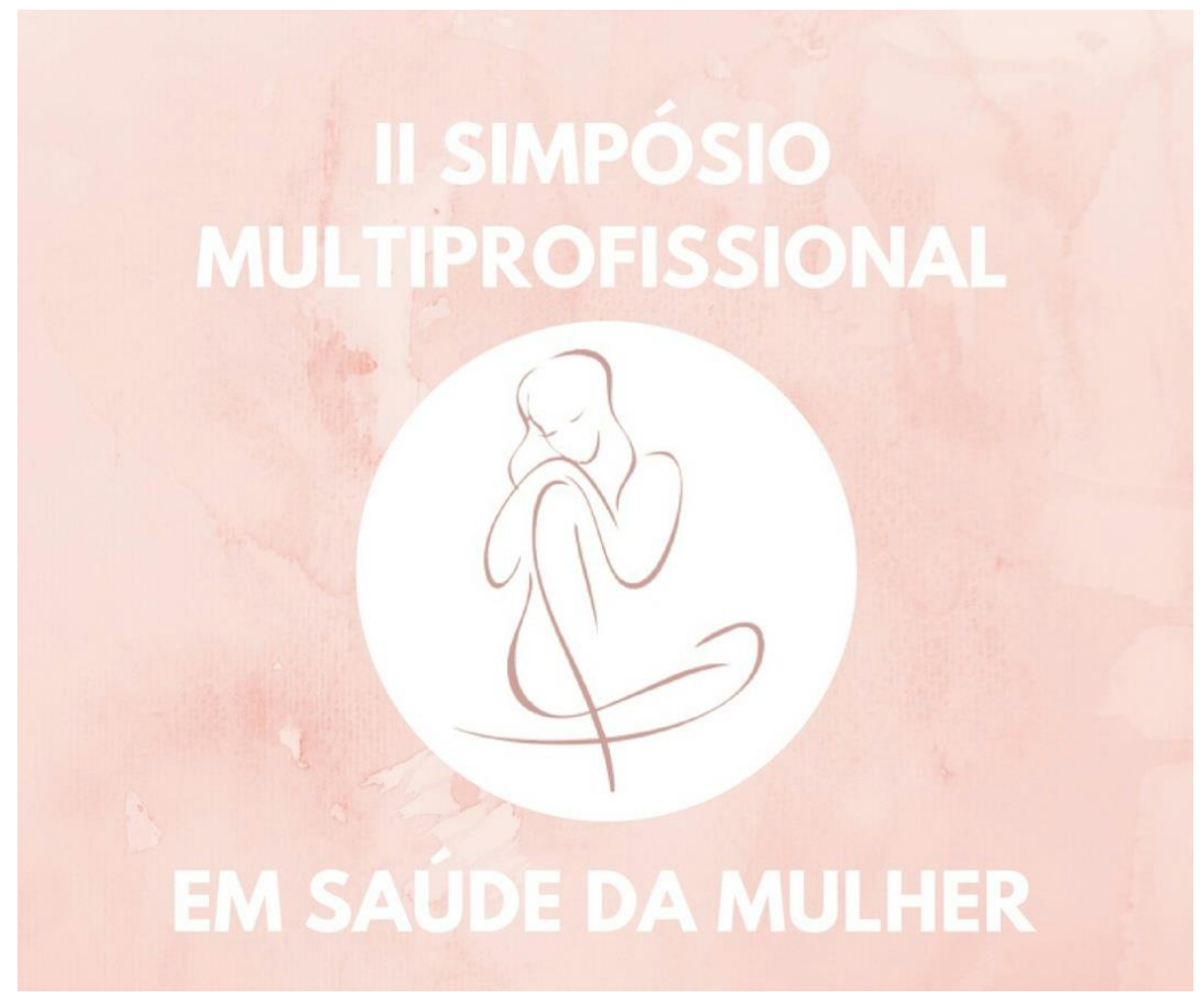

ANAIS DO II SIMPÓSIO MULTIPROFISSIONAL EM SAÚDE DA MULHER $2^{\circ}$ edição

Universidade Estadual do Piauí Teresina-PI

2019 


\section{FICHA TÉCNICA}

II SIMPÓSIO MULTIPROFISSIONAL EM SAÚDE DA MULHER

Realizado nos dias 22, 23 e 24 de Março de 2019

Local: Auditório da Universidade Estadual do Piauí - UESPI

\section{PROGRAMAÇÃO DO II SIMPÓSIO MULTIPROFISSIONAL EM SAÚDE DA MULHER}

\section{MINICURSOS}

- Análise de Lesões Perineais

- Atuação da Fisioterapia no pós-operatório imediato e tardio de lipoaspiração

- Pilates no Solo

- Os benefícios da atividade física para a saúde feminina

\section{PALESTRAS/MESAS REDONDAS}

- Fisioterapia no Trabalho de Parto - Dra Leide Cavalcante

- Síndrome da Zica Congênita - Dra Amarílis Loisa

- Parto Vacinal para Diabetes gestacional - Dra Hellen Gomes

- Pré-Natal Odontológico: da Gestante ao Neonato - Tereza Alcântara

- Resistência Metabólica e Emagrecimento em Mulheres - Ághata Crystian

- Sexualidade da Mulher Idosa: Educação em Saúde no Processo do Envelhecimento - Dra Cidiana

- Imunoterapia no tratamento do câncer de mama - Dra Ester Miranda

- Atuação da Estomaterapia na Saúde da Mulher - Dra Patrícia Cavalcanti

- Depressão Decorrentes de Mudanças Hormonais Feminina: Reconhecer e tratar é preciso - Dra Ana Maria Barros

- Mesa redonda - Feminicídio

- Mesa redonda - Sexualidade - Dra Madalena Carcará 
DOCENTES ORGANIZADORES

Nidiany Medeiros da Silva (Presidente Docente)

Christiane Lopes Xavier (Presidente da Comissão de Palestra)

Maura Cristina Porto Feitosa (Presidente da Comissão de Palestra)

Renata Pereira da Silva (Presidente da Comissão Científica)

\section{COMISSÃO ORGANIZADORA \\ Paulo Roberto Pereira Borges (Presidente Discente) \\ Kamylla Farias de Oliveira (Vice-Presidente Discente)}

Alan Jefferson Alves Reis

Ana Maria Silva Bílio

Bárbara Hélen Vieira e Silva Santos

Breno Alves da Silva

Camila Lima de Carvalho

Carla Valéria Gomes da Silva

Daniel dos Santos Nunes

Daniele Alves da Silva

Eva Karoline Rodrigues da Silva

Fábio Vinícius Ferreira Silva

Francisca Istefanne Santos Ricardo

Lauryanna de Queiroz Silva

Lílian Maria Magalhães Costa de Oliveira

Loyhara Ingryd Melo

Luís Eduardo de França Barros Menezes

Marcelane Macêdo dos Santos
Maria do Rosário de Fátima da Silva Rocha

Maria Yasmim da Conceição Chagas

Mariana Antonia de Carvalho Ferreira

Marília Cardoso Coelho

Marina Landa Ribeiro dos Santos Silva

Mayara Monteiro Andrade

Mayara Oliveira Ribeiro

Milena Alves de Araújo

Patrícia Torres da Silva

Pedro Henrique Moraes Mendes

Sarah Lays Campos da Silva

Thays Pereira da Rocha

Verônica Lorrânny Lima Araújo

Vitória Gabriele Barros de Araújo

Wanessa Lima Mendes

Yasmine Castelo Branco dos Anjos 


\section{COMISSÃO CIENTÍFICA}

Renata Pereira da Silva (Presidente Docente da Comissão Científica)

Ana Maria Silva Bílio (Presidente Discente da Comissão Científica)

Mayara Oliveira Ribeiro (Vice-Presidente Discente da Comissão Científica)

Alan Jefferson Alves Reis

Daniel dos Santos Nunes

Daniele Alves da Silva

Kamylla Farias de Oliveira

Lílian Maria Magalhães Costa de Oliveira

Maria do Rosário de Fátima da Silva Rocha

Patrícia Torres da Silva

Paulo Roberto Pereira Borges

Yasmine Castelo Branco dos Anjos

\section{AVALIADORES}

Priscila Figueiredo Cruz Ramos

Mariana Dantas Coutinho

Hengrid Graciely Nascimento Silva

Lucília da Costa Silva

Bárbara Carvalho dos Santos

Thyara Maria Stanley Vieira Lima

Edilene Rocha de Sousa

Lauryanna de Queiroz Silva

Kaíta Daniele Alves Soares

Cibelle de Sousa e Silva 


\section{COMENTÁRIO DO PRESIDENTE}

A saúde da mulher pode ser definida como as doenças ou condições que são exclusivas às mulheres ou envolvem diferenças sexuais particularmente importantes para as mulheres. Essa definição reconhece as crescentes evidências científicas que sustentam um enfoque direcionado para sexo e gênero, e expande o conceito de saúde da mulher para além da ênfase tradicional nos órgãos reprodutivos e suas funções.

A situação de saúde envolve diversos aspectos da vida, como a relação com o meio ambiente, o lazer, a alimentação e as condições de trabalho, moradia e renda. No caso das mulheres, os problemas são agravados pela discriminação nas relações de trabalho e a sobrecarga com as responsabilidades com o trabalho doméstico. Outras variáveis como raça, etnia e situação de pobreza realçam ainda mais as desigualdades.

As mulheres vivem mais do que os homens, porém adoecem mais frequentemente. Diante disso, este projeto objetivou discutir a atuação dos profissionais na saúde da mulher com o intuito de promover uma discussão sobre prevenção e cuidado feminino, além disso, compartilhar os conhecimentos dos profissionais para sanar dúvidas e possibilitar aos discentes, profissionais e docentes uma ampliação em sua formação e atuação respectivamente.

O II Simpósio Multiprofissional em Saúde da Mulher teve o apoio da Universidade Estadual do Piauí - UESPI e do Conselho Regional de Enfermagem do Piauí, agradecemos também toda a comissão organizadora pelo o empenho e dedicação, assim como todos os palestrantes, e os professores organizadores como a Presidente da Comissão Científica Renata Sampaio e a Presidente Docente Nidianny Medeiros. E, claro agradecemos à todos os inscritos pela magnifica participação nesse grande simpósio Multiprofissional, direcionado para profissionais e acadêmicos dos cursos da saúde, com ênfase nas ações de promoção, proteção, assistência e recuperação da saúde, executadas nos diferentes níveis de atenção à saúde, as discussões foram através de palestras, minicursos e mesasredondas.

\section{Paulo Roberto Pereira Borges}




\section{Sumário}

"VOU ME AMAR DE JANEIRO A JANEIRO": EDUCAÇÃO EM SAÚDE PARA A PROMOÇÃO DA SAÚDE MENTAL DA MULHER.

CONSIDERAÇÕES ACERCA DA SAÚDE DA MULHER PRIVADA DE LIBERDADE

EDUCAÇÃO EM SAÚDE COMO ESTRATÉGIA DE PREVENÇÃO DO CÂNCER DE MAMA E PROMOÇÃO DO AUTOCUIDADO.

A RODA DE CONVERSA COMO ESTRATÉGIA EDUCATIVA E PARTICIPATIVAPARA FALAR SOBRE A DOENÇA DE ALZHEIMER

RASTREAMENTO DE CÂNCER DE COLO DO ÚTERO: UM DESAFIO PARA A SAÚDE PÚBLICA .................. 16 SOFRIMENTO PSICOLÓGICO PROVOCADO EM MULHERES COM FIBROMIALGIA ......................................18 PRÁXIS DA FISIOTERAPIA NA PREVENÇÃO DA INCONTINÊNCIA URINÁRIA DURANTE E APÓS A GRAVIDEZ.

ATUAÇÃO FISIOTERAPÊUTICA NO TRATAMENTO DA DIÁSTASE ABDOMINAL EM MULHERES NO PÓSPARTO

AVALIAÇÃO DO ESTADO NUTRICIONAL E DA INGESTÃO DE CÁLCIO EM MULHERES NO CLIMATÉRIO

A SALA DE ESPERA COMO ESTRATÉGIA PARA PROMOÇÃO DO ALEITAMENTO MATERNO: RELATO DE EXPERIÊNCIA

EDUCAÇÃO EM SAÚDE SOBRE HANSENÍASE EM UM GRUPO DE MULHERES ............................................. 28

FATORES ASSOCIADO AO NEAR MISS MATERNO: REVISÃO BIBLIOGRÁFICA …….................................... 30

FATORES ASSOCIADOS À SÍNDROME HELLP EM PACIENTES INTERNADAS EM UTIS MATERNAS:

REVISÃO BIBLIOGRÁFICA

PERFIL DE MORBIMORTALIDADE POR NEOPLASIA MALIGNA DO COLO DO ÚTÉRO NO BRASIL DA ÚLTIMA DÉCADA.

EDUCAÇÃO EM SAÚDE SOBRE HIV/AIDS PARA MULHERES: RELATO DE EXPERIENCIA . 36 USO DA ESTIMULAÇÃO ELÉTRICA PARA A REDUÇÃO DA GORDURA CORPORAL DE MULHERES: REVISÃO DE LITERATURA. .38 EFEITOS DO MÉTODO PILATES SOBRE O EQUILÍBRIO DE IDOSAS: UMA REVISÃO BIBLIOGRAFICA .... 40 IMPACTOS DA ENDOMETRIOSE NA QUALIDADE DE VIDA DA MULHER .. .42 PERSPECTIVAS DA ATUAÇÃO FISIOTERAÊUTICA NO TRATAMENTO DO LINFEDEMA ASSOCIADO AO CÂNCER DE MAMA.

PESQUISA ETNOFARMACOLOGICA SOBRE O USO DE PLANTAS MEDICINAIS E FITOTERÁPICOS POR MULHERES NO AUTOCUIDADO

PERFIL EPIDEMIOLÓGICO DE CÂNCER DE COLO DE ÚTERO NO ESTADO DO PIAUÍ, NOS ANOS DE 2012 A 2016 


\title{
"VOU ME AMAR DE JANEIRO A JANEIRO": EDUCAÇÃO EM SAÚDE PARA A PROMOÇÃO DA SAÚDE MENTAL DA MULHER
}

\author{
Abimael de Carvalho 1; Sarah Lays Campos Da Silva2; Vivia Rhavena Pimentel \\ Costa3; Suellen Aparecida Patrício Pereira4;
}

${ }^{1}$ Universidade Estadual do Piauí, Teresina, Piauí

2 Universidade Estadual do Piauí, Teresina, Piauí;

${ }^{3}$ Universidade Estadual do Piauí, Teresina, Piauí;

${ }^{4}$ Especialista a nível de Residência em Saúde da Família e da Comunidade, Universidade Estadual do Piauí, Teresina, Piauí;

\section{E-mail do apresentador: abimaeldecarvalho123@gmail.com}

INTRODUÇÃO: De acordo com os dados de 2017 do ministério da saúde, o Brasil tem vivenciado um aumento das problemáticas relativas à saúde mental. A sociedade brasileira é a recordista latino-americana em casos de depressão, a campeã mundial em relação à ansiedade, e o quarto no crescimento das taxas de suicídio. Com base nessas indicações, a campanha janeiro branco, busca colocar o tema saúde mental em máxima evidência em nome da prevenção e do combate ao adoecimento emocional da população. (Cardoso E Galera, 2011). OBJETIVOS: Identificar o modo como a mulher vivencia o processo de envelhecimento, bem como as necessidades de cuidado que se apresentam nessa fase. MATERIAIS E MÉTODOS: Trata-se de um relato de experiência, vivenciado a partir de uma atividade de educação em saúde de um evento denominado "Vou me amar de janeiro a janeiro" realizado em alusão à campanha janeiro branco, desenvolvido pela equipe do Núcleo de Apoio a Saúde da Família do município de Barras, com o suporte do Programa Academia da Saúde. O evento ocorreu no dia 23 de janeiro de 2019, na praça Senador Joaquim Pires, no centro de Barras-PI, tendo início às $8 \mathrm{~h}$ e encerrando às $11 \mathrm{~h}$, contando com a presença de centenas de mulheres de todas as faixas etárias. À frente das atividades estavam enfermeiros, psicólogos, fisioterapeutas e educadores físicos, e dentre as ações desenvolvidas, destacam-se: distribuição de panfletos, camisas, fitas e balões brancos, dinâmicas, peças teatrais e roda de conversa. Além disso, foram disponibilizados vários serviços de saúde, como verificação de PA e, ainda, práticas integrativas como a auriculoterapia. RESULTADOS E DISCUSSÃO: Diante das ações executadas, percebeu-se a relevância do evento ao promover espaços de interação entre a equipe de saúde com o público feminino ao passo em que ouviam vivências e queixas e, a partir disso, apropriando-se do conceito de clínica ampliada, promoviam saúde em um ambiente diferenciado. Entretanto, notou-se que o tema ainda não é muito conhecido, e por gerar muito tabu em sua volta, merece mais evidência. Foi possível identificar transtornos comuns já evidenciados pela literatura, como a ansiedade e a depressão, sendo que por conta destes, outras queixas, como má qualidade do sono e dores, podem se fazer presente acarretando gastos com serviços de saúde. (BARBOSA, DIMENSTEIN \& LEITE, 2014). Contudo, no decorrer do evento foram adquirindo novos conhecimentos acerca da temática e reconhecendo a importância dos cuidados com a saúde emocional. Nesse sentido, entende-se que por meio da educação em saúde as mulheres adquiriram uma maior consciência da conjuntura atual em que vivem, e isso nos leva a pensar que o cuidado à saúde perpassa pelo olhar integral em relação ao contexto em que estão inseridas e aos aspectos de cada ciclo de vida. CONSIDERAÇÕES FINAIS: Através da ação realizada foi possível transmitir novos conhecimentos e repassar orientações acerca da temática, além de possibilitar reflexões a respeito de estratégias, como o uso 
de metodologias ativas no processo, como uma abordagem mais sensibilizada e acessível que pode ser implementada para uma melhor atenção à saúde desse público.

PALAVRAS-CHAVES: SAÚDE MENTAL; SAÚDE DA MULHER; PROMOÇÃO DA SAÚDE.

\section{REFERÊNCIAS:}

CARDOSO, L; GALERA, F. A. S; O cuidado em saúde mental na atualidade. Rev. Esc. Enfer USP, 2011; 45(3): 687-91.

BARBOSA, L. B.; DIMENSTEIN, M; LEITE, J. F; Mulheres, violência e atenção em saúde mental: questões para (re) pensar o acolhimento no cotidiano dos serviços. Av. Psicol. Latinoam. Bogotá, v. 32, n. 2, p. 309-320, Aug. 2014. 


\title{
CONSIDERAÇÕES ACERCA DA SAÚDE DA MULHER PRIVADA DE LIBERDADE
}

\author{
Alan Jefferson Alves Reis ${ }^{1}$; Pedro Henrique Moraes Mendes²; Nanielle Silva Barbosa³ \\ José Francisco Ribeiro ${ }^{4}$
}

${ }^{1}$ Universidade Estadual do Piauí - UESPI, Teresina, Piauí;

2Universidade Federal do Piauí - UFPI, Teresina, Piauí;

${ }^{3}$ Universidade Estadual do Piauí - UESPI, Teresina, Piauí;

${ }^{4}$ Mestre em Enfermagem. Universidade Estadual do Piauí - UESPI, Teresina, Piauí.

E-mail do apresentador: allanjeferson012@gmail.com

INTRODUÇÃO: Mulheres constituem percentual cada vez mais significativo na população privada de liberdade, apresentando, em sua maioria, situação de vulnerabilidade. Em relação ao atendimento à saúde dessa população, peculiaridades devem ser consideradas, tomando por base, diretrizes e princípios da saúde da mulher no âmbito do SUS. Diante da problemática de saúde vivenciada e a existência de uma diversidade de estratégias voltadas para este público este estudo sugere a compreensão do que vem sendo estudado com relação à saúde dessas mulheres. OBJETIVO: Analisar evidências científicas relacionadas à saúde da mulher privada de liberdade, respondendo a seguinte questão de pesquisa: o que apontam as evidências científicas em relação à saúde da mulher privada de liberdade? MATERIAIS E MÉTODOS: Trata-se de uma revisão integrativa da literatura com levantamento bibliográfico realizado entre dezembro de 2018 e fevereiro de 2019, aplicando-se os descritores, baseados na estratégia PICo: prisões, prisioneiros e saúde da mulher às bases de dados Literatura Latino-Americana e do Caribe em Ciências da Saúde (LILACS), Banco e Dados em Enfermagem (BDENF) e Medical Literature Analysis and Retrieval Sistem Online (MEDLINE/Pubmed). Incluiu-se estudos primários disponíveis na integra, desenvolvidos com seres humanos, ensaios clínicos randomizados controlados individuais, pesquisas quase-experimental, transversais, Iongitudinais e opinião de especialistas. Utilizou-se instrumento elaborado pelos autores para extração de dados relevantes. Classificou-se as produções conforme nível de evidência. RESULTADOS E DISCUSSÃO: A busca resultou em oitenta e três produções, das quais, vinte e três foram incluídas como amostra. Destacou-se o ano de 2016, com seis publicações. Dezoito artigos eram internacionais. Quanto à metodologia, treze estudos eram quantitativos. Onze produções classificaram-se com nível de evidência 5. Originaram-se cinco categorias temáticas: Patologias e comportamentos de mulheres privadas de liberdade; Percepções de mulheres privadas de liberdade em relação à saúde; Saúde sexual e reprodutiva de mulheres privadas de liberdade; Reintegração social de mulheres privadas de liberdade; Saúde mental de mulheres privadas de liberdade. CONSIDERAÇÕES FINAIS: Pode-se indicar como limitação deste estudo o fato da busca de artigos ter sido realizada apenas em três bases de dados, sendo possível que alguns artigos relacionados não tenham sido incluídos na revisão. Ressalta-se que as bases utilizadas são as mais comumente consultadas. Conclui-se que mulheres privadas de liberdade são mais vulneráveis a agravos à saúde do que a população feminina em geral, onde há o acesso limitado aos cuidados de saúde, muitas vezes prestados indevidamente. Relata-se predominantemente agravos relacionados às comorbidades, comportamentos de risco, saúde sexual e reprodutiva e saúde mental. Deve-se considerar as condições sociais como relevantes. Instiga-se a elaboração, implementação e monitoramento de políticas públicas destinadas 
à esta população, consideradas indispensáveis para o aprimoramento das condições de saúde das mulheres em cárcere, além do pleno envolvimento ético e qualificação de profissionais que atuam no cuidado direto a estas pessoas.

PALAVRAS-CHAVE: Prisões; Prisioneiros; Saúde da Mulher.

\section{REFERÊNCIAS:}

BRAUNSTEIH, H.R. Mulher encarcerada: trajetória entre a indignação e o sofrimento por atos de humilhação e violência. 2015

MARTINS, J.S. A sociedade visto abismo: novos estudos sobre exclusão, pobreza e classes sociais. 2016. 


\title{
EDUCAÇÃO EM SAÚDE COMO ESTRATÉGIA DE PREVENÇÃO DO CÂNCER DE MAMA E PROMOÇÃO DO AUTOCUIDADO
}

\author{
Aline Tavares Gomes ${ }^{1}$; Jaciane Santos Marques²; Marilyse de Oliveira Meneses ${ }^{3}$; Socorro \\ Adriana de Sousa Meneses Brandão ${ }^{4}$; Samira Rêgo Martins de Deus Leal ${ }^{5}$. \\ 1Pós- Graduanda no programa de Residência Multiprofissional em Saúde da Família e \\ Comunidade da Universidade Estadual do Piauí - UESPI, Teresina, Piauí; \\ 2 Pós- Graduanda no programa de Residência Multiprofissional em Saúde da Família e \\ Comunidade da Universidade Estadual do Piauí - UESPI, Teresina, Piauí; \\ ${ }^{3}$ Pós- Graduanda no programa de Residência Multiprofissional em Saúde da Família e \\ Comunidade da Universidade Estadual do Piauí - UESPI, Teresina, Piauí; \\ ${ }^{4}$ Mestre. Preceptora do Programa da Residência Multiprofissional em Saúde da Família e \\ Comunidade da Universidade Estadual do Piauí - UESPI, Teresina, Piauí; \\ ${ }^{5}$ Doutora. Preceptora do Programa da Residência Multiprofissional em Saúde da Família \\ e Comunidade da Universidade Estadual do Piauí - UESPI, Teresina, Piauí.
}

E-mail do apresentador: alinettavaresg@gmail.com

INTRODUÇÃO: O câncer de mama é classificado como o tipo mais comum de câncer entre a s mulheres do mundo e do Brasil, responsável por cerca de $28 \%$ dos casos novos a cada ano. O conhecimento da mulher em relação ao seu corpo é extremamente importante para a detecção precoce, pois a maior parte dos cânceres de mama é descoberto pelas próprias mulheres. Nesse contexto, o diagnóstico precoce configura-se como a melhor estratégia no âmbito da prevenção, este podendo ser realizado através da mamografia, exame clínico das mamas (ECM) e a radiografia das mamas. Tendo a prática sistemática do autoexame das mamas, na maioria dos casos, o ponto de partida para o início desse diagnóstico. A educação em saúde configura-se como uma proposta político-pedagógica que procura proporcionar a melhoria da atenção à saúde, por meio da prevenção de doenças, como também, estimulando a participação da população por meio de ações educativas. OBJETIVO: Relatar a experiência de residentes de enfermagem da Residência Multiprofissional em Saúde da Família e Comunidade (RMSFC) da Universidade Estadual do Piauí (UESPI) em uma ação educativa sobre câncer de mama realizada com um grupo de mulheres em alusão ao outubro rosa. MATERIAIS E MÉTODOS: Trata-se de um estudo descritivo na modalidade de relato de experiência realizado no mês de outubro de 2018, desenvolvido pela Residência Multiprofissional em Saúde da Família e Comunidade (RMSFC) em um centro de convivência pertencente à área de abrangência de uma Unidade Básica de Saúde (UBS) localizada em um bairro da zona sul no município de Teresina/PI tendo como público alvo o grupo de mulheres participantes de um projeto de práticas corporais intitulado "MoviMente", além de mulheres moradoras do bairro. RESULTADOS E DISCUSSÃO: A ação educativa desenvolvida sobre a temática foi realizada no dia "D" em alusão ao outubro rosa. Após a realização de uma caminhada temática pelo bairro desenvolveu-se uma gincana em saúde mediante a utilização de metodologias ativas em saúde. As participantes foram separadas em dois grupos e as enfermeiras residentes conduziram a atividade por meio da realização de perguntas disparadoras direcionadas aos dois grupos com o objetivo de facilitar a interação das participantes na atividade. Os aspectos abordados na gincana foram: o que é o câncer de mama, fatores de risco, sinais e sintomas, forma de detecção, tratamento e autoexame das mamas. Na ocasião foi possível que as enfermeiras retirassem dúvidas sobre o assunto, demonstrassem como é 
feito o autoexame das mamas e ratificassem a importância de sua realização, além de proporcionar espaço para que mulheres já acometidas pela patologia relatassem sua experiência dirimindo assim pré-conceitos e enfatizando a importância do autocuidado. CONSIDERAÇõES FINAIS: Pode-se perceber que ações de promoção à saúde como esta, realizada através de metodologias ativas, proporcionam maior descontração e consequentemente maior

espaço de socialização e aprendizado, dirimindo timidez e proporcionando maior vínculo com os profissionais de saúde, sendo importante que os profissionais se apropriem dessas metodologias que proporcionam maior assimilação de conhecimentos de temáticas relacionadas à saúde.

PALAVRAS-CHAVE: Educação em Saúde, Neoplasias da mama, Atenção Primária a Saúde.

\section{REFERÊNCIAS:}

BRASIL. Ministério da Saúde. Departamento de Atenção Básica. Controle dos cânceres do colo do útero e da mama - 2. ed. - Brasília : Editora do Ministério da Saúde, 2013.

SOARES, Lidia Santos et al. Conhecimento de mulheres sobre medidas de detecção precoce do câncer de mama. HU Revista, v. 43, n. 2, p. 127-132, 2017.

BRASIL. Ministério da Saúde. Instituto Nacional de Câncer. Câncer de mama: é preciso falar disso. Rio de Janeiro: Inca, 2014. 


\section{A RODA DE CONVERSA COMO ESTRATÉGIA EDUCATIVA E PARTICIPATIVAPARA FALAR SOBRE A DOENÇA DE ALZHEIMER}

Aline Tavares Gomes ${ }^{1}$; Jaciane Santos Marques²; Marilyse de Oliveira Meneses ${ }^{3}$; Socorro
Adriana de Sousa Meneses Brandão ${ }^{4}$; Samira Rêgo Martins de Deus Leal ${ }^{5}$.

1Pós- Graduanda no programa de Residência Multiprofissional em Saúde da Família e Comunidade da Universidade Estadual do Piauí - UESPI, Teresina, Piauí;

${ }^{2}$ Pós- Graduanda no programa de Residência Multiprofissional em Saúde da Família e Comunidade da Universidade Estadual do Piauí - UESPI, Teresina, Piauí;

3Pós- Graduanda no programa de Residência Multiprofissional em Saúde da Família e Comunidade da Universidade Estadual do Piauí - UESPI, Teresina, Piauí;

${ }^{4}$ Mestre. Preceptora do Programa da Residência Multiprofissional em Saúde da Família e Comunidade da Universidade Estadual do Piauí - UESPI, Teresina, Piauí;

${ }^{5}$ Doutora. Preceptora do Programa da Residência Multiprofissional em Saúde da Família e Comunidade da Universidade Estadual do Piauí - UESPI, Teresina, Piauí.

E-mail do apresentador: alinettavaresg@gmail.com

INTRODUÇÃO: O Alzheimer é uma doença neuro-degenerativa que provoca o declínio das funções cognitivas, reduzindo as capacidades de trabalho e relação social e interferindo no comportamento e na personalidade da pessoa, constituindo a causa mais comum de demência - um grupo de distúrbios cerebrais que causam a perda de habilidades intelectuais e sociais. Na doença de Alzheimer, as células cerebrais degeneram e morrem, causando um declínio constante na memória e na função mental. Embora existam casos esporádicos em pessoas de 50 anos e a prevalência na faixa etária de 60 a 65 anos esteja abaixo de 1\%, a partir dos 65 anos ela praticamente duplica a cada cinco anos. Depois dos 85 anos de idade, atinge 30 a $40 \%$ da população, O risco é mais alto em pessoas que têm história familiar de Alzheimer ou outras demências. Para quem chegou aos 65 anos, o risco futuro de surgir Alzheimer é de 12\% a $19 \%$ no sexo feminino; e de $6 \%$ a $10 \%$ nos homens. OBJETIVO: Relatar a experiência de residentes de enfermagem da Residência Multiprofissional em Saúde da Família e Comunidade (RMSFC) da Universidade Estadual do Piauí (UESPI) em uma ação educativa sobre a doença de Alzheimer, realizada com um grupo de mulheres em alusão ao fevereiro roxo. MATERIAIS E MÉTODOS: Trata-se de um estudo descritivo na modalidade de relato de experiência realizado no mês de fevereiro de 2019, desenvolvido pela Residência Multiprofissional em Saúde da Família e Comunidade (RMSFC) em uma Unidade Básica de Saúde (UBS) localizada em um município próximo a Teresina, onde é desenvolvido parte da extensão de categoria de enfermagem, um dos requisitos da residência. Teve como público alvo um grupo de mulheres que aguardava para serem atendidas no dia dedicado a cuidar da saúde das mulheres na referida UBS. RESULTADOS E DISCUSSÃO: A atividade educativa foi desenvolvida através de uma sala de espera conduzida pelas preceptoras e residentes. As participantes foram convidadas a participar de uma roda de conversa, onde após a entrega de tarjetas com tópicos sobre o assunto tais como: o que é o Alzheimer? Quais os sinais e sintomas? Como se dá o tratamento?Como podemos prevenir? Qual o tratamento? Quem é mais acometido?. Na ocasião, elas eram estimuladas a ler suas tarjetas e responder de acordo com seus conhecimentos prévios e experiências já vivenciadas. A intenção do uso dessa metodologia era deixar a roda mais participativa, e elas se sentirem parte importante do processo de discussão. Foi estimulado também a participação das demais participantes no decorrer das respostas. Logo após essa chuva de respostas, as preceptoras e as residentes enfatizaram mais sobre as perguntas realizadas, elucidando dúvidas e 
equívocos mencionados na chuva de respostas. CONSIDERAÇÕES FINAIS: Pode-se perceber que ações de educação em saúde que estimulam a participação ativa do participante, bem como o seu conhecimento prévio sobre o tema, proporcionam maior descontração, socialização e aprendizado das partes envolvidas, participantes e condutores da atividade. O vínculo é fortalecido e o objetivo é mais bem alcançado.

PALAVRAS-CHAVE: Educação em Saúde, Doença de Alzheimer, Atenção Primária a Saúde.

\section{REFERÊNCIAS:}

AREIAS, B. B; BONFIM, M. M. D; SCHIAVETO, F. V. A participação da enfermagem frente ao cuidador de idosos portadores de Alzheimer. Revista Fafibe On-Line, Bebedouro SP, v. 8, n. 1, p. 44-63, 2015.

POIRIER, J; GAUTHIER, S. Doença de Alzheimer: o guia completo. MG Editores, 2016. 


\title{
RASTREAMENTO DE CÂNCER DE COLO DO ÚTERO: UM DESAFIO PARA A SAÚDE PÚBLICA
}

\author{
Antonio Lucas Mendes Araújo1, Irene Sousa da Silva²
}

${ }^{1}$ Acadêmico de Medicina da Universidade Estadual do Maranhão, campus Caxias - MA 2 Mestre em Ciências e saúde, Docente da Universidade Estadual do Maranhão, campus Caxias - MA

E-mail do apresentador: antoniolma33@gmail.com

INTRODUÇÃO: O Câncer do Colo do Útero (CCU) é definido como uma neoplasia que costuma ter crescimento lento, que pode durar muitos anos, sendo caracterizado como neoplasia maligna a partir da descoberta de alterações nas células epiteliais do colo uterino. No Brasil, apresenta uma incidência anual de 16.340 casos sendo o terceiro mais frequente, e a quarta causa de mortalidade de mulheres. O Maranhão é o segundo estado com maior incidência. No município de Caxias, este tipo de câncer é o segundo em incidência em mulheres, perdendo somente para o câncer mamário. A forma primaria de prevenção ao CCU se faz com a diminuição do risco de contagio do Papiloma Vírus Humano, e de forma secundaria, com o rastreamento realizado pelo exame citopatológico, que analisa os tipos celulares presentes na cérvice uterina. No Brasil, é a partir da Atenção Primária a Saúde (APS) que o rastreamento é realizado, e o mesmo está estruturado em um programa específico baseado na base populacional, onde a mulher é vinculada a Estratégia de Saúde da Família. OBJETIVOS: Avaliar a eficácia do rastreio de câncer do colo do útero em uma Unidade Básica de Saúde (UBS), do município de Caxias-MA. MATERIAIS E MÉTODOS: Estudo transversal, realizado a partir da análise de prontuários de uma UBS do município de Caxias- MA, cujas variáveis estudadas foram: idade, taxa de cobertura do exame citopatológico, tempo entre a realização e resultado do exame e tipo de resultado a partir da leitura da lâmina. RESULTADOS E DISCUSSÃO: Das 889 mulheres com idade entre 25 a 65 anos, correspondente à área adescrita da UBS em estudo, 297(33,40\%) realizaram o exame citopatológico, aproximadamente $50 \%$ destas, apresentaram idade entre 25 a 45 anos. Do total de mulheres que submeteram-se à realização do exame, somente 121 (40,74\%) obtiveram o resultado, a maioria, com mais de 30 dias após a coleta do material. Dos achados colposcópicos 03 (2,48\%) foram positivos para neoplasia intraepitelial cervical de nível acentuado e todas realizam biópsia. Dos achados microbiológicos: garderella $s p$ 11 (9,16\%), chlamydia sp 04 (3,33\%) e lactobacillus sp 106 (87,51\%). A taxa de cobertura foi satisfatória, conforme as diretrizes do Ministério da Saúde, entretanto, a eficiência do rastreio foi prejudicada, pelo número de exames sem obtenção de resultados, e o tempo superior ao estimado pelo protocolo do Ministério da Saúde. CONSIDERAÇÕES FINAIS: A UBS em análise atingiu a meta de cobertura, entretanto, a não eficiência do andamento dos resultados pode comprometer o diagnóstico e tratamento em tempo oportuno e ocasionar o aumento da prevalência e mortalidade por câncer de colo do útero.

PALAVRAS-CHAVES: Câncer do colo do útero, Unidade básica de saúde, eficácia. 


\section{REFERÊNCIAS:}

DE SOUZA, Suzy Anne Lopes; DA SILVEIRA, Lia Márcia Cruz. (Re) Conhecendo a Escuta como Recurso Terapêutico no Cuidado à Saúde da Mulher. Revista Psicologia e Saúde, v. 11, n. 1, p. 42, 2019.

DE SENNA, Patricia Alves; DA FONSECA, Rosa Maria Godoy Serpa. CLUBE DE MÃES: ESPAÇO PARA INTERVENÇÃO EM SAÚDE DA MULHER. Revista da Escola de Enfermagem da USP, v. 29, n. 1, p. 34-46, 2018. 


\title{
SOFRIMENTO PSICOLÓGICO PROVOCADO EM MULHERES COM FIBROMIALGIA
}

\author{
SOUSA, Dalila Marielly ${ }^{1}$;LOPES, Caroline Sousa²; CARCARA, Lorena Rocha de \\ Abrantes $^{3}$; AQUINO, Maria Luiza da Silva ${ }^{4}$; REIS, Mayra Dayananda Cunha ${ }^{5}$; ROCHA, \\ Silvana Santiago da ${ }^{6}$ \\ ${ }^{1}$ Universidade Federal do Piauí-UFPI, Teresina, Piauí; \\ 2 Universidade Federal do Piauí-UFPI, Teresina, Piauí; \\ 3 Universidade Federal do Piauí-UFPI, Teresina, Piauí; \\ 4 Universidade Federal do Piauí-UFPI, Teresina, Piauí; \\ 5 Universidade Federal do Piauí-UFPI, Teresina, Piauí; \\ 6 Pós-doutorado em Enfermagem, Universidade Federal do Piauí-UFPI, Teresina, Piauí; \\ E-mail do apresentador: dalilamarielly24@gmail.com
}

INTRODUÇÃO: Segundo a Sociedade Brasileira de Reumatologia a fibromialgia é a doença reumatológica mais frequente tendo como característica dor musculoesquelética difusa e crônica afetando, em sua maioria, mulheres entre 40 e 55 anos. Essa síndrome tem um impacto negativo na qualidade de vida dessas pessoas resultando em estresse psicológico podendo desencadear até depressão e ansiedade. OBJETIVOS: Identificar através das produções literárias o impacto negativo no psicológico de mulheres com fibromialgia. MATERIAIS E MÉTODOS: Revisão integrativa da literatura, realizada em dezembro e janeiro de 2018 e 2019. Para seleção dos artigos utilizou-se bases de dados de literatura Latino-Americano e de Caribe em ciências da saúde (LILACS); Scientific Eletronic Library Online (SCIELO). Foram critérios de inclusão artigos em inglês e português, com textos completos, nos anos de 2015 a 2018, com os descritores: Fibromialgia, Qualidade de vida, Depressão. Foram encontrados 90 dos quais 18 atenderam aos critérios de inclusão que constituem a amostra final. RESULTADOS E DISCUSSÃO: A relação entre as emoções e os sintomas físicos provavelmente contribui para os diversos fatores que favorecem a evolução da fibromialgia. Por isso as interrelações entre os sistemas biológicos, psicológicos e sociais são importantes pois influenciam os processos de saúde e doença. Interesse e dedicação para melhorar o apoio social nesses pacientes parecem ser um componente crucial a ser incluído na prática para melhorar a qualidade da saúde dessa população. CONSIDERAÇÕES FINAIS: Através dessa revisão de literatura concluímos a necessidade de mais estudos relacionados a fibromialgia, seus sintomas e impactos psicossociais nas mulheres, que na maioria dos casos apresentam sintomas de depressão e ansiedade além da qualidade de vida prejudicada pelas incapacidades relacionadas a doença. Logo, é necessário métodos próprios da psicologia para tratar essas implicações, que devem ser vistas com seriedade pelos profissionais e parentes da paciente.

PALAVRAS-CHAVES: Fibromialgia, Qualidade de vida, Depressão. 


\section{REFERÊNCIAS:}

FREITAS, Rodrigo Pegado de Abreu; ANDRADE, Sandra Cristina; SPYRIDES, Maria Helena; MICUSSI, Maria Thereza A.B.; SOUSA, Maria Bernadete C. Impactos do apoio social sobre os sintomas de mulheres brasileiras com fibromialgia. Ver. Bras. Reumatol. Rio Grande do Norte, 2017;57(3):197-203.

COELHO, Charlotte. Ansiedade e depressão na fibromialgia. Psicologia. pt. Porto, 2016.

GOULART, Rubens; PESSOA, Cinthia; JUNIOR, Império Lombardi. Aspectos psicológicos da síndrome da fibromialgia juvenil: revisão de literatura. Ver. Bras. Reumatol. São Paulo, 2018;56(1):69-74. 


\title{
PRÁXIS DA FISIOTERAPIA NA PREVENÇÃO DA INCONTINÊNCIA URINÁRIA DURANTE E APÓS A GRAVIDEZ
}

\author{
${ }^{1}$ Francisca Clara Lopes Soares; ${ }^{2}$ Thele Albuquerque da Silva; ${ }^{3}$ Marcelino Martins
}

\begin{abstract}
${ }^{1}$ Graduanda de Fisioterapia pela Universidade Estadual do Piauí - UESPI, Teresina, PI; ${ }^{2}$ Graduanda de Fisioterapia pela Universidade Estadual do Piauí - UESPI, Teresina, PI; ${ }^{3}$ Doutor em Engenharia Biomédica, Universidade Estadual do Piauí - UESPI, Teresina, $\mathrm{PI}$;
\end{abstract}

E-mail do apresentador: claralopes234@gmail.com

INTRODUÇÃO: A incontinência urinária (IU) está entre as doenças mais prevalentes na população feminina. A gravidez é um dos principais fatores causais da IU em mulheres, devido a processos fisiológicos sequenciais que ocorrem durante a gestação e o parto que resultam na diminuição da função muscular do assoalho pélvico (FMAP). $O$ início dela ocorre frequentemente durante a gravidez ou no pós-parto com 30 a 50\% de mulheres afetadas. O tratamento fisioterapêutico tem se mostrado promissor na prevenção da IU entre as mulheres. Atualmente, a Sociedade Internacional de Continência (ICS) aponta a reabilitação do assoalho pélvico (AP) como sendo a primeira opção de tratamento da IU, uma vez que os exercícios perineais são de baixo custo e risco, não invasivos, combatem a diminuição da FMAP. OBJETIVO: Identificar na literatura a atuação da fisioterapia na prevenção da incontinência urinária durante e após a gravidez, baseado na literatura mais atual. MÉTODOS: Trata-se de uma revisão sistemática realizada a partir de consulta às bases de dados eletrônicas Pubmed e PEDro, utilizando os descritores incontinência urinária, fisioterapia e gravidez. Foram incluídos ensaios clínicos randomizados, na língua portuguesa inglesa ou espanhola dos últimos anos, com o intuito de verificar o que recentemente tem sido estudado como tratamento preventivo da IU. A pesquisa resultou em 57 artigos, após análise, oito estudos clínicos foram selecionados, após análise por dois revisores independentes e aplicação dos critérios de elegibilidade guiados por meio de ficha padronizada. A qualidade metodológica dos artigos foi avaliada através da escala PEDro. RESULTADOS E DISCUSSÃO: Dentre os nove artigos analisados, foi possível observar que as principais intervenções utilizaram exercícios para o assoalho pélvico os quais podem aumentar a força muscular pélvica e prevenir o surgimento da incontinência urinária. Para o treinamento de força foi utilizado a performance de Kegel, manual de orientação de exercícios domiciliares (MOED) e exercícios de contração máxima, contração rápida e sustentada. Os mesmos promoveram resultados no aumento da força muscular pélvica com consequente continência de urina. CONSIDERAÇÕES FINAIS: Conclui-se que a atuação fisioterapêutica é de suma importância para prevenção da incontinência urinária, principalmente devido ao treinamento dos músculos do assoalho pélvico que traz resultados também no tratamento dessa disfunção durante e após a gravidez.

PALAVRAS-CHAVE: Incontinência urinária, fisioterapia, gravidez.

\section{REFERÊNCIAS:}

ASSIS, L.C. et al. (2015). Effectiveness of an illustrated home exercise guide on promoting urinary continence during pregnancy: a pragmatic randomized trial. Brazilian Journal of Gynecology and Obstetrics, 37 (10), 460-466. 
FRITEL, X. et al (2015). Prevention of urinary incontinence with previous prenatal pelvic floor exercises. Obstetrics \&Gynecology, 126 (2), 370-377.

KO, P. C. et al. (2010). A randomized controlled study of prenatal pelvic floor exercises to prevent and treat urinary incontinence. International Journal of Urogynecology, 22 (1), 1722.

SANGS, B. et al. (2016). Is a 6-week pelvic floor muscle exercise program supervised and effective in preventing end-stage stress urinary incontinence in primiparous women: a randomized controlled trial. European Journal of Obstetrics and Gynecology and Reproductive Biology, 197, 103-110.

BOTELHO, S. M. et al. (2015). Abdominal-pelvic kinesiotherapy for muscle training of the pelvic floor: a proposal tested in different groups. International Journal of Urogynecology, 26 (12), 1867-1869.

SUT, K. H. et al. (2015). Effect of pelvic floor muscle exercise on pelvic floor muscular activity and voiding functions during pregnancy and postpartum period. Neurourology and Urodynamics, 35 (3), 417-422.

STAFNE, S. et al. (2012). Does regular exercise, including pelvic floor muscle training, prevent urinary and anal incontinence during pregnancy? A randomized controlled trial. BJOG: An International Journal of Obstetrics and Gynecology, 119 (10), 1270-1280. 


\section{ATUAÇÃO FISIOTERAPÊUTICA NO TRATAMENTO DA DIÁSTASE ABDOMINAL EM MULHERES NO PÓS-PARTO}

${ }^{1}$ Francisca Clara Lopes Soares; ${ }^{2}$ Thele Albuquerque da Silva; ${ }^{3}$ Marcelino Martins;

${ }^{1}$ Graduanda de Fisioterapia pela Universidade Estadual do Piauí - UESPI, Teresina, PI; ${ }^{2}$ Graduanda de Fisioterapia pela Universidade Estadual do Piauí - UESPI, Teresina, PI; ${ }^{3}$ Doutor em Engenharia Biomédica, Universidade Estadual do Piauí - UESPI, Teresina, $\mathrm{PI}$;

E-mail do apresentador: claralopes234@gmail.com

INTRODUÇÃO: A diástase abdominal é definida como uma deficiência com separação da linha média dos músculos retos abdominais ao longo da linha alba. Condição essa altamente prevalente no pós-parto devido ao período de progressão da gravidez, no qual há mais alongamento dos músculos abdominais, perda do vetor de força e, talvez, diminuição da força de contração. Um dos objetivos da fisioterapia aplicada a esta etapa é promover uma estimulação da musculatura, em particular abdominal e pélvica, para melhorar a sua tonicidade. OBJETIVO: Identificar na literatura a forma de atuação do profissional fisioterapeuta no tratamento da diástase abdominal em mulheres no pós-parto. MÉTODOS: Trata-se de uma revisão sistemática realizada a partir de consulta às bases de dados eletrônicas Pubmed e PEDro, utilizando os descritores diástase muscular, músculos abdominais e fisioterapia. Foram incluídos ensaios clínicos randomizados, na língua portuguesa inglesa ou espanhola. A pesquisa resultou em 29 artigos, após análise por dois avaliadores independentes, 9 estudos clínicos foram selecionados de acordo com os critérios de elegibilidade determinados. A qualidade metodológica dos artigos foi avaliada através da escala PEDro. RESULTADOS: Dentre os nove artigos analisados, foi possível observar aplicação de treinamento de força, estimulação elétrica neuromuscular, exercícios somados a kinesio taping e método Pilates caracterizando técnicas fisioterapêuticas, que trouxeram resultados positivos com relação a diminuição da diástase abdominal e também a força muscular. CONSIDERAÇÕES FINAIS: A assistência fisioterapêutica no pós-parto reduz significativamente a diástase abdominal, além de promover a estimulação e força dos músculos, o que contribui também na prevenção de disfunções secundárias a diástase.

PALAVRAS-CHAVES: Diástase muscular, fisioterapia, músculos abdominais.

\section{REFERÊNCIAS:}

GLUPPE, S.L. et al.(2018). In order to obtain a postpartum evaluation program in a randomized and controlled clinical trial. Physiotherapy 98 (4), 260-268.

KAMEL, D.M. et al. (2017). Neuromuscular Electrical Stimulation and Restoration of Abstinence Muscle Strength of Post Nasal Dysthesis. Annals of Rehabilitation Medicine, 41 (3), 465.

GÜRŞEN, C. et al. (2015). Effects of exercise and Kinesio taping on abdominal recovery in women with cesarean section: a randomized controlled pilot study. Archives of Gynecology and Obstetrics, 293 (3), 557-565 
VASSELJEN, O. \& FLADMARK, A.(2010). Thickness of abdominal muscle contraction and function after specific and general exercises: A randomized controlled trial in patients with chronic low back pain. Manual therapy, 15 (5), 482-489.

GIACOMINI, M.B. et al.(2016). The Pilates method increases the strength and performance of the respiratory muscles as well as the thickness of the abdominal muscle. Journal of Bodywork e Movement Therapies, 20 (2), 258-264. 


\title{
AVALIAÇÃO DO ESTADO NUTRICIONAL E DA INGESTÃO DE CÁLCIO EM MULHERES NO CLIMATÉRIO
}

\author{
Fernanda do Nascimento Araújo 1; Patrícia Barbosa Pereira²; Camila Guedes Borges de \\ Araújo3.
}

${ }^{1}$ Universidade Federal do Piauí, Teresina, Piauí;

2 Universidade Federal do Piauí, Teresina, Piauí;

${ }^{3}$ Mestre, Universidade Federal do Piauí, Teresina, Piauí;

E-mail do apresentador: fernanda151927@hotmail.com

INTRODUÇÃO: O climatério é um termo que se refere à interrupção perdurável da menstruação sendo evidenciada pelas modificações hormonais, o qual atinge mulheres entre 40 a 65 anos de idade. Entre as principais doenças que surgem são a osteoporose e as doenças cardiovasculares, estando relacionadas ao aumento da gordura visceral, inatividade física, inadequado consumo de cálcio, e a qualidade da dieta. Estima- se que 1 em cada 4 mulheres tenham osteoporose, após a menopausa- uma doença silenciosa e mal diagnosticada, que afeta 200 milhões de mulheres no mundo. O cálcio dietético, é necessário para prevenção da osteoporose, corresponde a $99 \%$ dos ossos e dentes, também é necessário para a transmissão dos impulsos nervoso, e, vale destacar que a vitamina $D$ é essencial na regulação do metabolismo do cálcio. OBJETIVOS: Avaliar, através de uma revisão literária, o estado nutricional e o nível de ingestão do cálcio por mulheres no climatério e conhecer seus efeitos para saúde. MATERIAIS E MÉTODOS: Foi realizada uma revisão integrativa- narrativa, através da busca de artigos pesquisados nas bases de dados SciELO e BVS, encontrando-se aproximadamente 234.275 resultados. Foram incluídos artigos que abordassem o estado nutricional e o consumo alimentar de mulheres adultas na fase do climatério, resultando em amostra com 20 artigos. RESULTADOS E DISCUSSÃO: Pode-se observar que as mulheres climatéricas possuem o ensino fundamental e médio completo, renda de 1 a 2 salários mínimos, e estado de sobrepeso ou obesidade. Dentre as doenças presentes, destacou- se a HA, dislipidemias, DM, artralgia, hipertireoidismo e osteopenia. Quanto á ingestão alimentar, as mulheres apresentaram baixo consumo de micronutrientes, tais como cálcio (<1200 mg / d) e vitamina $D(<10 \mathrm{mcg} / \mathrm{d})$, além das fibras, que apresentaram valores inferiores a $25 \mathrm{~g} / \mathrm{d}$, e elevado consumo de macronutrientes tais como proteína ( $>35 \%$ do VET) e carboidratos (>65 \% do VET). CONSIDERAÇÕES FINAIS: Conclui- se que as mulheres climatéricas encontram - se em excesso de peso e alimentam-se de forma inadequada quanto ao cálcio, mas também à proteína e vitamina $\mathrm{D}$, os quais seriam fundamentais para prevenir doenças como a osteoporose.

PALAVRAS-CHAVES: climatério, cálcio, estado nutricional, menopausa. 


\section{REFERÊNCIAS:}

CRUZ, R. A; et. al. Aspectos nutricionais e dietéticos em mulheres climatéricas. Revista de atenção a saúde, 2017.

PASSOS, S. P; TOMÉ F. M; REIS, M. J. A. Levantamento da conduta alimentar e fatores de risco para o surgimento da osteoporose em mulheres no climatério. J Health Sci Inst. 2017.

LIMA, L. F; et. al. Perfil do consumo alimentar e da relação cálcio/proteína de mulheres no climatério. Nutr. clin. diet. hosp. 2016. 


\title{
A SALA DE ESPERA COMO ESTRATÉGIA PARA PROMOÇÃO DO ALEITAMENTO MATERNO: RELATO DE EXPERIÊNCIA
}

\author{
Jaciane Santos Marques ${ }^{1}$; Aline Tavares Gomes²; Marilyse de Oliveira Meneses ${ }^{3}$; Socorro \\ Adriana de Sousa Meneses Brandão4; Samira Rêgo Martins de Deus Leal ${ }^{5}$.
}

1 Pós- Graduanda no programa de Residência Multiprofissional em Saúde da Família e Comunidade da Universidade Estadual do Piauí - UESPI, Teresina, PI;

${ }^{2}$ Pós- Graduanda no programa de Residência Multiprofissional em Saúde da Família e Comunidade da Universidade Estadual do Piauí - UESPI, Teresina, PI;

${ }^{3}$ Pós- Graduanda no programa de Residência Multiprofissional em Saúde da Família e Comunidade da Universidade Estadual do Piauí - UESPI, Teresina, PI;

${ }^{4}$ Mestre. Preceptora do Programa da Residência Multiprofissional em Saúde da Família e Comunidade da Universidade Estadual do Piauí - UESPI, Teresina, PI;

${ }^{5}$ Doutora. Preceptora do Programa da Residência Multiprofissional em Saúde da Família e Comunidade da Universidade Estadual do Piauí - UESPI, Teresina, PI.

E-mail do apresentador: jacianesantosmarques@hotmail.com

INTRODUÇÃO: O aleitamento materno é considerado a estratégia de maior impacto na redução de mortalidade infantil. É uma prática fundamental para a promoção de saúde das crianças, pois fornece do ponto de vista nutricional o que há de melhor em macronutrientes e micronutrientes nos aspectos quantitativos e qualitativos. Além disso, a amamentação, mais do que um meio de alimentação, é o primeiro contato físico entre mãe e filho, e também uma das principais formas de fortalecer este importante vínculo familiar e de amor nos primeiros meses de vida do bebê. OBJETIVOS: Relatar as percepções obtidas por enfermeiras residentes sobre uma sala de espera com o tema aleitamento materno para gestantes. MATERIAIS E MÉTODOS: Trata-se de um estudo descritivo do tipo relato de experiência vivenciado por residentes de enfermagem da Residência Multiprofissional em Saúde da Família e Comunidade (RMSFC) da Universidade Estadual do Piauí (UESPI) a cerca da importância da sala de espera para gestantes, antes da consulta de pré- natal realizada no mês de agosto de 2018, em uma Unidade Básica de Saúde (UBS) localizada em um bairro da zona sul no município de Teresina/PI. RESULTADOS E DISCUSSÃO: Para a operacionalização da sala de espera, a mesma foi dividida em momentos. No primeiro momento foi realizado um acolhimento por meio de um Bom Dia acolhedor para recepcionar os usuários que chegavam à UBS para o atendimento. No segundo momento, para o desenvolvimento da temática foi realizado um mural do conhecimento com o uso de imagens ilustrativas sobre a temática, abordando os seguintes aspectos: o que é aleitamento materno; composição do leite materno; importância e benefícios do aleitamento materno; dificuldades na amamentação e pega adequada do bebê. No terceiro momento, e como forma de avaliar o entendimento dos usuários presentes sobre a explanação do conteúdo, foi utilizado um envelope contendo mitos e verdades em que cada participante da sala de espera, retirava um papel desse envelope, realizava a leitura e as residentes esclareciam os questionamentos levantados. CONSIDERAÇÕES FINAIS: Houve uma intensa participação com esclarecimento de muitas duvidas acerca do tema, representando um grande aprendizado para todos os participantes. Desta forma, percebe- se que a utilização das salas de espera na UBS é uma ferramenta fundamental para 0 
desenvolvimento da Educação em Saúde além de aproximar a comunidade dos profissionais e humanizar o cuidado.

PALAVRAS-CHAVE: Aleitamento Materno, Saúde da mulher, Promoção de saúde.

\section{REFERÊNCIAS:}

ALMEIDA, J. M.; LUZ, S. A. B.; UED, F.V. Apoio ao aleitamento materno pelos profissionais de saúde: revisão integrativa da literatura. Rev. Paul. Pediatria. 33 (3):355362, 2015

NUNES, L.M. Importância do aleitamento materno na atualidade. Boletim Científico de Pediatria, 4 (3) 3, 2015. 


\section{EDUCAÇÃO EM SAÚDE SOBRE HANSENÍASE EM UM GRUPO DE MULHERES}

Jaciane Santos Marques ${ }^{1}$; Aline Tavares Gomes²; Marilyse de Oliveira Meneses ${ }^{3}$; Socorro Adriana de Sousa Meneses Brandão4; Samira Rêgo Martins de Deus Leal ${ }^{5}$.

1 Pós- Graduanda no programa de Residência Multiprofissional em Saúde da Família e Comunidade da Universidade Estadual do Piauí - UESPI, Teresina, PI;

2 Pós- Graduanda no programa de Residência Multiprofissional em Saúde da Família e Comunidade da Universidade Estadual do Piauí - UESPI, Teresina, PI;

${ }^{3}$ Pós- Graduanda no programa de Residência Multiprofissional em Saúde da Família e Comunidade da Universidade Estadual do Piauí - UESPI, Teresina, PI;

${ }^{4}$ Mestre. Preceptora do Programa da Residência Multiprofissional em Saúde da Família e Comunidade da Universidade Estadual do Piauí - UESPI, Teresina, PI;

${ }^{5}$ Doutora. Preceptora do Programa da Residência Multiprofissional em Saúde da Família

e Comunidade da Universidade Estadual do Piauí - UESPI, Teresina, PI.

E-mail do apresentador: jacianesantosmarques@hotmail.com

INTRODUÇÃO: A hanseníase é uma doença infecciosa e crônica de grande relevância para a saúde pública, devido ao seu poder de causar incapacidades físicas, social e econômica, possuindo ações exclusivas voltadas para a sua eliminação em âmbito nacional por meio do Programa de Controle da Hanseníase, presente na Atenção Primária à Saúde (APS), em particular nas Equipes de Saúde da Família (EqSF), atendendo a população por meio de ações preventivas e curativas. A educação em Saúde configura-se como uma proposta político- pedagógica que procura proporcionar a melhoria da atenção à saúde, por meio da prevenção de doenças, como também estimulando a participação da população por meio de ações educativas. OBJETIVOS: Relatar as experiências e percepções obtidas por enfermeiras residentes em atividades educativas sobre a temática Hanseníase para mulheres em alusão ao janeiro roxo. MATERIAIS E MÉTODOS: Trata-se de um estudo descritivo na modalidade de relato de experiência realizado nos meses de Janeiro e fevereiro de 2019 por residentes de enfermagem da Residência Multiprofissional em Saúde da Família e Comunidade (RMSFC) da Universidade Estadual do Piauí (UESPI) com um grupo de mulheres participantes de um projeto de práticas corporais intitulado "MoviMente" desenvolvido no polo de academia da saúde da área de abrangência de uma Unidade Básica de Saúde (UBS) localizada em um bairro da zona sul no município de Teresina/PI. Além do desenvolvimento da atividade no grupo de mulheres a atividade educativa também foi replicada em diversas ocasiões, como em salas de espera na referida UBS. RESULTADOS E DISCUSSÃO: Foram realizadas ações educativas sobre a Hanseníase abordando a doença, sua forma de detecção, transmissão, sinais e sintomas, tratamento e como conviver com pacientes com hanseníase. Para um dialogo mais próximo das usuárias utilizou- se de linguagem direta e coloquial para melhor entendimento, uso de folders e envelopes contendo perguntas norteadoras como instrumentos facilitadores da roda de conversa. Durante a ação educativa, percebeu- se o interesse dessa população em relação ao tema, devido às dúvidas que surgiram no decorrer da ação, sendo estas esclarecidas no momento. CONSIDERAÇÔES FINAIS: A possibilidade de estar próximo à comunidade por meio de ações educativas, permite a criação de um vínculo maior entre usuários e profissionais de saúde que atuam na UBS. Desta forma, percebeu- se a importância da abordagem desta temática, pois permitiu esclarecimentos e desmistificações de questões acerca da doença.

PALAVRAS-CHAVE: Educação em Saúde, Hanseníase, Atenção Primária a Saúde. 


\section{REFERÊNCIAS:}

LEAL, D. R. et al. Programa de Controle da Hanseníase: uma avaliação da implantação no nível distrital. Saúde debate, Rio de janeiro, v. 41, n. Especial, p. 209-228, 2017.

Brasil. Ministério da Saúde. Secretaria de Vigilância em Saúde. Departamento de Vigilância das Doenças Transmissíveis. Guia prático sobre a hanseníase [recurso eletrônico] / Ministério da Saúde, Secretaria de Vigilância em Saúde, Departamento de Vigilância das Doenças Transmissíveis. - Brasília : Ministério da Saúde, 2017. 


\title{
FATORES ASSOCIADO AO NEAR MISS MATERNO: REVISÃO BIBLIOGRÁFICA
}

\author{
Laércio Bruno Ferreira Martins; Bárbara Carvalho dos Santos²
}

${ }^{1}$ Acadêmico, Universidade Estadual do Piauí-UESPI, Teresina, PI;

${ }^{2}$ Fisioterapeuta, Universidade Estadual do Piauí, Teresina-PI.

\section{E-mail do apresentador: laerciom42@gmail.com}

INTRODUÇÃO: De acordo com a Organização Mundia da Saúde (OMS), Near Miss ocorre quando uma mulher sobrevive a complicações ocorridas durante a gestação, parto ou puerpério. Esta condição ocorre até 100 vezes mais que os óbitos maternos. A prevalência de near miss varia amplamente; na América Latina encontra-se entre 0,34-4,92\%, dependendo dos critérios de definição utilizados. Entretanto, apesar de não existir consenso sobre os critérios para uma definição estrita de near miss, o conjunto das diversas definições pode ser útil para diversos objetivos, incluindo monitorização, vigilância epidemiológica e auditoria de cuidados de saúde, e recentemente tais definições tem sido utilizados para avaliar a assistência prestada nas maternidades. OBJETIVOS: Verificar os fatores associados ao Near Miss materno em pacientes internadas em UTIs maternas. MATERIAIS E MÉTODOS: Trata-se de uma revisão bibliográfica, na qual uma pesquisa sobre o tema foi realizada nas bases de dados Pubmed, SciELO, SpringerLink, Free Medical Journals e banco de dados Bireme, no período de dezembro de 2018 a fevereiro de 2019, com os descritores: Near Miss, Morbidade Materna, Complicações na Gravidez e UTI, nos idiomas português, inglês e espanhol. RESULTADOS E DISCUSSÃO: Foram encontrados 12 estudos, dos quais oito foram incluídos e quatro excluídos, de acordo com os critérios de inclusão e exclusão. A maioria dos estudos buscou descrever a incidência, a prevalência e o perfil clínico-epidemiológico das pacientes internadas na UTI. Os estudos associaram o near miss a partos cesáreos prévios, primiparidade e nuliparidade, gestação gemelar, pré-natal inadequado, um estudo associou o near miss a pré-natal em serviços públicos, peregrinação superior a uma hora, indução do parto, uso de fórceps, cesariana sem trabalho de parto, histórico de aborto e concepto natimorto e ter hipertensão, doenças hematológicas, cardiopatias, asma, diabetes ou epilepsia prévias à gravidez. Dentre as características sociodemográficas, foram fatores de risco maior densidade familiar, idade entre 10 e 14 anos e maior que 35, raça parda, preta ou indígena, ser solteira ou divorciada, baixa escolaridade e renda familiar. As complicações mais evidentes foram distúrbios hipertensivos como eclâmpsia, pré-eclâmpsia grave e Síndrome HELLP, infecções como endometrite, pneumonia, urinária, pancreatite e sepse, além de descolamento prematuro placentário, rotura uterina, placenta prévia, acretismo placentário, distúrbios hemorrágicos, tromboembólicos e cardíacos. CONSIDERAÇÕES FINAIS: O near miss materno foi associado a diversos fatores como primiparidade, realização inadequada do pré-natal, indução do parto, distúrbios hipertensivos, hemorrágicos e infecciosos, além do acometimento maior de mulheres em situação sociodemográfico desfavorável. Entretanto, embora possa ser considerado um forte preditor da assistência materna, é necessário que novas pesquisas sejam realizadas abordando o tema, tendo em vista a dificuldade de identificar um caso devido à extrema inespecificidade e dificuldade paramétrica acerca dos critérios a serem levados em conta no diagnóstico de um near miss e a diversidade de situações que podem induzir tal condição.

PALAVRAS-CHAVE: Near Miss; Morbidade Materna; Complicações na Gravidez; Unidade de Terapia Intensiva. 


\section{REFERÊNCIAS:}

CECATTI, J. C.; et.al.Maternal Near Miss among women using the public health system in the Amazon and Northeast regions of Brazil. Rev.Panam.Salud.Publica.,v.37,n.4,p.:232238,2015 .

DIAS, M. A. B.; etal. Incidence of maternal Near Miss in hospital childbirth and post partum: data from the Birth in Brazil study. Cad.Saúde Pública,v.30,p.:1-12,2014.

MORSE, M. L.; etal. Severe Maternal Morbidity and Near Misses in a Regional Reference Hospital.Rev.Bras.Epidemiol.,v.14,n.02,p.:310-322,2011.

NAKAMURA-PEREIRA, M.; etal. The Hospital Information Systemof the Brazilian Unified National Health System: a performance evaluation for auditing maternal Near Miss. Cad. Saúde Pública, v.29,n.07,p.:1333-1345,2013.

OLIVEIRA, L. C.; COSTA, A. A. R.. Óbitos fetais e neonatais entre casos de Near Miss materno. Rev. Assoc. Med. Bras.,v.59,n.05,p.:487-494,2013.

Maternal Near Miss in the intensive careunit: clinical and epidemiological aspects. Rev. Bras. Ter. Intensiva.,v.27,n.03,p.:220-227,2015.

ROSENDO, T. M. S. S.; RONCALLI,A.G.Prevalence and factors associated with Maternal Near Misses: a survey of the population in a capital city of the Brazilian Northeast. Ciência\&Saúde Coletiva, v.20,n.04,p.:1295-1304,2015.

RUDEY, E. L.; CORTEZ, L. E. R.; YAMAGUCHI, M. U. Identificação de Near Miss Materno em Unidadede Terapia Intensiva. Revista Saúde e Pesquisa, v.10,n.01,p.:145-155,2017. 


\title{
FATORES ASSOCIADOS À SÍNDROME HELLP EM PACIENTES INTERNADAS EM UTIS MATERNAS: REVISÃO BIBLIOGRÁFICA
}

\author{
Laércio Bruno Ferreira Martins"; Bárbara Carvalho dos Santos²
}

1 Acadêmico, Universidade Estadual do Piauí-UESPI, Teresina, PI; 2 Fisioterapeuta, Universidade Estadual do Piauí, Teresina-PI;

\section{E-mail do apresentador: laerciom42@gmail.com}

INTRODUÇÃO: A Síndrome HELLP (HELLP) é caracterizada por hemólise, aumento de enzimas hepáticas e plaquetopenia. Na maior parte dos casos está associada à préeclâmpsia, eclâmpsia e disfunção hepática durante a gestação. Essa síndrome ocorre em aproximadamente uma a duas mulheres a cada 1.000 gestações, em 4 a $12 \%$ das gestantes com pré-eclâmpsia grave e em até $11 \%$ das gestantes com eclâmpsia. A maioria dos casos acomete mulheres com gestação entre a $28^{\underline{a}}$ a $36^{\underline{a}}$ semanas, e no geral representa um quadro grave dentre as doenças hepáticas que acometem a gravidez, por oferecer alto risco de mortalidade materna e perinatal, oscilando entre $1 \%$ e $24 \%$ dos casos, sendo os óbitos decorrentes em $80 \%$ das vezes de complicações do sistema nervoso central. OBJETIVO: Verificar os fatores associados à Síndrome HELLP em pacientes internadas em UTIs maternas. MATERIAIS E MÉTODOS: Trata-se de uma revisão bibliográfica, na qual uma pesquisa sobre o tema foi realizada nas bases de dados Pubmed, SciELO, SpringerLink, Free Medical Journals e banco de dados Bireme, no período de dezembro de 2018 a fevereiro de 2019, com os descritores: HELLP, Pré-eclâmpsia e UTI, nos idiomas português, inglês e espanhol. RESULTADOS E DISCUSSÃO: Foram encontrados 11 estudos, dos quais sete foram incluídos e quatro excluídos, de acordo com os critérios de inclusão e exclusão. A maioria dos estudos buscou descrever a incidência e o perfil clínico-epidemiológico das pacientes internadas em UTIs. Nestes estudos, a HELLP esteve associada à primiparidade, antecedentes de cesariana, histórico familiar de distúrbios hipertensivos independentes ou associados a outras patologias, presença de diabetes mellitus, idade inferior a 21 anos, feto do sexo feminino, consumo de psicoativos e uso de álcool, presença de sobrepeso prévio à gravidez, distúrbios dislipidêmicos e ter feito fertilização in vitro. Foram apontados ainda como fatores associados dependência econômica, baixo nível de escolaridade e ser chefe de família. Um estudo associou a presença da síndrome ao tempo dispendido até a chegada a uma instituição obstétrica e outro estudo associou a presença da síndrome a mulheres com ovários policísticos. Dentre as principais complicações estavam o desenvolvimento de insuficiência cardíaca, insuficiência renal aguda, ruptura hepática, coagulopatias, distúrbios hemorrágicos como hemoperitônio e esteatose aguda gestacional. Como fatores protetores da síndrome foram evidenciados gravidez ou aborto prévios e ter tido pré-eclâmpsia em uma gestação anterior. CONSIDERAÇÕES FINAIS: Para a confecção deste material foi realizada uma extensa busca na literatura. Contudo evidenciou-se que ainda há poucos estudos referentes ao tema. Apesar de ser uma situação pouco conhecida e de rara ocorrência, os resultados obtidos demonstram a necessidade de mais pesquisas sobre o tema visto que trata-se de uma síndrome multifatorial estando relacionada muitas vezes a gestações precoces e primíparas, entre outros, sendo esta responsável por muitas complicações podendo levar até ao óbito. 
PALAVRAS-CHAVE: Pré-Eclâmpsia; Síndrome HELLP; Unidade de Terapia Intensiva.

\section{REFERÊNCIAS:}

CURIEL-BALSERA, E.; et al. Análisis de La morbimortalidad materna de lãs pacientes com pré eclampsia grave, eclampsia y síndrome HELLP que ingresan em uma Unidad de Cuidados Intensivos gineco-obstétrica. Med. Intensiva, v.35,n.08,p.:478-483,2011.

ENGJOM,H.M.; et al. Risk of eclampsia or HELLP-syndrome by institution availability and place of delivery - a population-based cohort study. Pregnancy Hypertension, v.14,p.:1-14,2018.

GUIMARÃES, N. M.; FERNANDES, E. S. Esteatose hepática aguda da gestação: relato de caso. HU Revista, v. 43, n.02,p.:179-182,2017.

MARTÍNEZ-CASTELLÓN, N.; FERNÁNDEZ-ORDÓÑEZ, E.; SÁNCHEZ-RUIZ, P. Management of HELLP syndrome in preterm pregnancy. Matronas Prof.,v.19,n.02,p.:1720,2018.

OLIVEIRA, L. C.; COSTA, A. A. R. Maternal near miss in the intensive careunit: clinical and epidemiological aspects. Rev. Bras. Ter. Intensiva, v.27,n.03,p.:220-227,2015.

PÉREZ, A. D.; et al. Interaction and dynamics of these risk factors in hypertensive disorders of pregnancy: a pilot study. Salud Uninorte, v. 33, n.01,p.:27-38,2017.

SCHNEIDER, D.; et al. The Association of Polycystic Ovary Syndrome and Gestational Hypertensive Disordersina Diverse Community-Based Cohort. Journal of Pregnancy,v.2019,p.:1-6,2019. 


\title{
PERFIL DE MORBIMORTALIDADE POR NEOPLASIA MALIGNA DO COLO DO ÚTÉRO NO BRASIL DA ÚLTIMA DÉCADA
}

\author{
Maria Camila Leal de Moura1; Renata Pereira da Silva1; Verônica Lorrany Lima Araújo1; \\ Gisele Lopes Cavalcante ${ }^{2}$
}

${ }^{1}$ Centro Universitário Santo Agostinho - UNIFSA, Teresina, PI

${ }^{2}$ Mestranda em Ciências Farmacêuticas pela Universidade Federal do Piauí - UFPI,

Teresina, PI

E-mail do apresentador: camilaleal.cw7@hotmail.com

INTRODUÇÃO: O câncer de colo do útero passou a ocupar um lugar de destaque nas taxas de morbimortalidade entre as mulheres de todo o mundo, afetando principalmente às de baixos níveis econômicos, constituindo assim, na contemporaneidade, a principal causa de óbitos por neoplasia entre a população feminina que vivem em países em processo de desenvolvimento. No Brasil, estimativas do Instituto Nacional do Câncer (INCA) apontam que o câncer de colo uterino seja a terceira neoplasia maligna mais ocorrente no sexo feminino e a quarta causa de morte de mulheres por câncer no país, acresce que sua ocorrência tem-se dado de forma extensiva e crescente. OBJETIVO: O presente estudo objetivou avaliar o perfil de morbimortalidade por neoplasia maligna do colo do útero no Brasil a fim de corroborar na compreensão desse agravo de saúde pública. MÉTODOS: Constitui-se de um estudo documental, retrospectivo e de série temporal baseado em dados coletados por meio Morbidade Hospitalar do SUS (SIH/SUS) do Departamento de Informática do Sistema Único de Saúde (DATASUS). Analisou-se a tendência de não completude segundo ano de diagnóstico, região, faixa etária, raça/cor e valores dos serviços hospitalares no recorte temporal de Janeiro de 2008 a Dezembro de 2018 em consonância com a 10 $10^{\text {a }}$ revisão da versão brasileira da Classificação Internacional de Doenças (CID BR-10). RESULTADOS E DISCUSSÃO: Demonstrou-se que desde o ano de 2008 as taxas de incidência mantêm-se constante ficando por cerca de vinte mil casos de internações hospitalares no SUS a cada ano da série temporal apontando que há necessidade de uma intensa intervenção a nível nacional em programas de prevenção dos casos para diminuição dessas taxas. Observou-se que o grupo etário de maior prevalência foi de 40 a 49 anos indo ao encontro com estudos de etiologia da neoplasia. No que diz respeito as regiões, o Sudeste apresentou maior ocorrência com 39,01\% ( $n=95285)$ por conseguinte o Nordeste com 27,71\% ( $n=67797)$ e o Sul com 20,39\% ( $n=49903)$ casos de internações. No que concerne a raça/cor os resultados demonstraram maior incidência na branca com 36,68\% ( $n=90131)$ seguidos de parda com 35,89\% ( $n=87708)$ e preta com $4,77 \%(n=115575)$. No tocante aos valores provenientes dos serviços hospitalares para atendimento dos casos têm-se uma ascensão onde em 2008 tinha-se um gasto de $R$ \$ 15.620.657,64 passando para $\mathrm{R} \$ 33.200 .111,33$ em 2018. CONSIDERAÇÕES FINAIS: Mediante a avaliação infere-se que a morbimortalidade pelo câncer de colo de útero no Brasil como um agravo com taxas de incidência constantes e geram altíssimos gastos públicos particularmente em faixa etária acima de 40 anos, desse modo as análises das variáveis do presente estudo visam corroborar na implementação de ações, estratégias e políticas governamentais voltadas para a integralidade à feminina e consequente minimização dessa problemática de saúde pública. 
PALAVRAS-CHAVE: Neoplasia maligna do colo do útero, Câncer, Saúde Pública, Internações Hospitalares.

\section{REFERÊNCIAS:}

Ministério da Saúde (BR). Secretaria Nacional de Assistência à . Saúde, Instituto Nacional do Câncer- INCA. Câncer no Brasil: dados dos registros de câncer de base populacional. Rio de Janeiro(RJ); 2018. 


\title{
EDUCAÇÃO EM SAÚDE SOBRE HIV/AIDS PARA MULHERES: RELATO DE EXPERIENCIA
}

\author{
${ }^{1}$ Marilyse de Oliveira Meneses ${ }^{1}$;Aline Tavares Gomes ${ }^{2}$; Jaciane Santos Marques ${ }^{3}$; Socorro \\ Adriana de Sousa Meneses Brandão ${ }^{4}$; Samira Rêgo Martins de Deus Leal ${ }^{5}$. \\ ${ }^{1}$ Pós- Graduanda no programa de Residência Multiprofissional em Saúde da Família e \\ Comunidade da Universidade Estadual do Piauí - UESPI, Teresina, Piauí; \\ 2 Pós- Graduanda no programa de Residência Multiprofissional em Saúde da Família e \\ Comunidade da Universidade Estadual do Piauí - UESPI, Teresina, Piauí; \\ ${ }^{3}$ Pós- Graduanda no programa de Residência Multiprofissional em Saúde da Família e \\ Comunidade da Universidade Estadual do Piauí - UESPI, Teresina, Piauí; \\ ${ }^{4}$ Mestre. Preceptora do Programa da Residência Multiprofissional em Saúde da Família e \\ Comunidade da Universidade Estadual do Piauí - UESPI, Teresina, Piauí; \\ ${ }^{5}$ Doutora. Preceptora do Programa da Residência Multiprofissional em Saúde da Família \\ e Comunidade da Universidade Estadual do Piauí - UESPI, Teresina, Piauí.
}

E-mail do apresentador: marilyse_meneses@hotmail.com

INTRODUÇÃO: O vírus da imunodeficiência adquirida (HIV) destrói os mecanismos de defesa do corpo e permite que doenças oportunistas se instalem nele. Historicamente, a infecção pelo HIV tem adquirido um novo padrão quanto à evolução clínica e ao perfil epidemiológico. No Brasil a epidemia distingue-se em fases. A primeira caracterizada por homens homossexuais de elevada escolaridade, atribuindo-se o conceito de "grupos de risco". Na segunda fase, assumiu-se o conceito de "comportamento de risco", devido à contaminação por uso de drogas injetáveis, e a terceira e atual fase, pela elevada incidência no sexo feminino, com baixa escolaridade, admitindo-se o conceito de "vulnerabilidade". Esta última, vivenciada por mulheres, pode ser compreendida em três dimensões: a individual, decorrente da dificuldade de acesso às informações, a social, resultante do acesso a serviços públicos; e, por último, a política, determinada pela elaboração de políticas de saúde. OBJETIVO: Relatar as experiências e percepções obtidas em atividades de promoção à saúde sobre HIV para mulheres na Zona Sul de Teresina- Piauí. MATERIAS E MÉTODOS: Estudo descritivo do tipo relato de experiência, realizado por meio de rodas de conversa que abordavam ações de prevenção à infecção pelo HIV. As atividades foram executadas no mês de Dezembro de 2018, por residentes de enfermagem da Residência Multiprofissional em Saúde da Família e Comunidade (RMSFC), com mulheres capturadas na sala de espera de duas unidades básicas de saúde localizadas na Zona Sul de Teresina, enquanto aguardavam atendimento médico e odontológico. RESULTADOS: As ações propostas foram desenvolvidas utilizando metodologias ativas de ensino-aprendizagem, oportunizando o desenvolvimento da autonomia dos indivíduos como sujeitos ativos do processo educativo. As atividades foram desenvolvidas em quatro momentos e em todos eles realizaram-se as seguintes ações: Inicialmente optou-se por uma dinâmica de acolhimento, logo após, utilizando um varal expositivo, realizou-se a entrega de imagens que denotavam situações ou condições em que se poderia ou não contrair o vírus HIV, tais como: picada por inseto, amamentação, abraço, sexo sem preservativo, amamentação, gestação, beijo, espirro, compartilhamento de talheres, seringas e vaso sanitário. E ao final procedeu-se a avaliação do momento. Observou-se que embora as participantes reconhecessem questões referentes à diferenciação entre a infecção pelo HIV e o desenvolvimento da Síndrome da Imunodeficiência Adquirida (AIDS), 
ainda persistiam falas carregadas de preconceito em decorrência do desconhecimento das medidas de prevenção e contágio. Notou-se que a picada do inseto, o compartilhamento de talheres, vaso sanitário e espirro ainda eram apontados por muitas como causa da infecção. Notadamente verificou-se que possuíam o conhecimento acerca da transmissão pelo sexo sem preservativo, assim como pelo compartilhamento de seringas e durante 0 período gestacional. No que tange a amamentação e beijo percebeu-se uma divisão de opiniões acerca do contágio, esclarecidos após explicação. CONSIDERAÇÕES FINAIS: Diante da atual epidemia da AIDS, tida um grave problema de saúde pública e da recente mudança no perfil clínico e epidemiológico, faz-se necessário intensificar o desenvolvimento de atividades de prevenção e promoção à saúde com vistas a instrumentalizar os indivíduos enquanto agentes ativos do seu processo saúde- doença.

PALAVRAS-CHAVE: HIV, Promoção em saúde, Controle das doenças transmissíveis.

\section{REFERÊNCIAS:}

MOURA, J.P; FARIA, M.R. Caracterização e perfil epidemiológico das pessoas que vivem com HIV /AIDS. Revista de Enfermagem da UFPE on line, Recife, 11(Supl. 12), p. 5214-20, 2017.

CAMPOS, C. G. A. P, ESTIMA, S. L, SANTOS, V. S, LAZZAROTTO, A. R. Vulnerability to HIV in adolescents: a retrospective study at a counseling and testing center. Revista Mineira de Enfermagem, v. 18, n. 2, p. 315-9, 2014.

SOUSA, M.C.P; SANTO, A.C.G.E; MOTTA, S.K.A. Gênero, Vulnerabilidade das Mulheres ao HIV/Aids e Ações de Prevenção em Bairro da Periferia de Teresina, Piauí, Brasil. Revista Saúde e Sociedade, São Paulo, v. 17, n.2, p.58-68, 2008 


\title{
USO DA ESTIMULAÇÃO ELÉTRICA PARA A REDUÇÃO DA GORDURA CORPORAL DE MULHERES: REVISÃO DE LITERATURA
}

\author{
Monaliza de Sousa Moura1; Mayara Andrade Monteiro²; Mayra Andrade Monteiro; \\ Marcelino Martins ${ }^{4}$; \\ 1,2 Universidade Estadual do Piauí,Teresina,Piauí; \\ ${ }^{3}$ Curso Politécnico,Teresina,Piauí; \\ ${ }^{4}$ Mestre, Universidade Estadual do Piauí,Teresina,Piauí;
}

E-mail do apresentador: monalizasousamoura@hotmail.com

INTRODUÇÃO: Segundo dados de 2015 da Associação Brasileira para o Estudo da Obesidade e da Síndrome Metabólica, quase $60 \%$ dos brasileiros estão acima do peso. Além dos riscos à saúde física, essa característica também interfere na saúde mental e provoca, muitas vezes, problemas de aceitação com o corpo. Ainda de acordo com esse estudo, a obesidade acomete mais mulheres do que homens e o acúmulo de gordura abdominal também foi mais frequente nesse sexo, sendo também as mulheres com maiores queixas em relação aos acúmulos de gordura corporal (BARBALHO et al., 2007). Considerando esses aspectos, métodos são constantemente pesquisados a fim de auxiliar na redução de gordura, dentre eles, os métodos de eletroestimulação- forma de tratamento não invasiva e amplamente utilizada por fisioterapeutas. OBJETIVOS: Analisar a eficiência da estimulação elétrica na redução da gordura corporal de mulheres. MATERIAIS E MÉTODOS: Constitui uma revisão de literatura, realizada no mês de fevereiro de 2019, por meio de consulta as bases de dados eletrônicas Periódicos Capes e Scielo, com os descritores: lipólise, adiposidade, estimulação elétrica, gordura e fisioterapia, com os seus respectivos em inglês e associados ao operador booleano AND. Foram incluídos: artigos em português e inglês, entre os anos de 2010 a 2019, que investigaram acerca da utilização da estimulação elétrica, através de qualquer aparelho, para reduzir a gordura corporal em mulheres de qualquer faixa etária. Foram excluídos estudos incompletos, fora do recorte temporal, duplicados nas bases, editoriais, revisões, que incluíam homens e sem intervenção. RESULTADOS E DISCUSSÃO: Foram encontrados 62 artigos e incluídos cinco no estudo por encaixarem-se nos critérios estabelecidos. Dentre os mais recentes, encontram-se o de Aroca et al. (2017) e Leite et al. (2017). O primeiro investigou o efeito da eletrolipólise na adiposidade abdominal e de flancos; como resultado observou diferença estatística entre os grupos intervenção e controle na reavaliação imediata, após 15 minutos de aplicação da técnica e na reavaliação final, contudo as variáveis antropométricas não modificaram significativamente. O segundo avaliou a influência da associação da criolipólise e radiofrequência no tratamento da adiposidade no abdômen inferior; constatou redução significativa das médias adipométricas quando comparadas ao prétratamento, porém as variáveis IMC, peso e perimetria não modificaram significativamente. Anteriormente, Carollo et al. (2013) já buscava relatar a aplicação de ultrassom associado à corrente elétrica estereodinâmica na adiposidade abdominal e normalização do perfil lipídico.Verificou-se redução nas medidas antropométricas, na mensuração após a quinta sessão, porém, as medidas iniciais retornaram ao final do estudo. No perfil lipídico, os níveis de colesterol total melhoraram efetivamente. Além destes, Macedo et al. (2013) e Melo et al. (2012) também demonstraram resultados 
satisfatórios na redução de medidas após a aplicação de estimulação elétrica. CONSIDERAÇÕES FINAIS: A estimulação elétrica é um método com resultados positivos para redução da gordura corporal em mulheres. Todavia, são necessários novos estudos, com amostras maiores para comprovar efetivamente a diminuição de gordura, pois após o procedimento, há grandes chances de a gordura reaparecer. Assim, para ser eficaz também a longo prazo, deve ser combinado uma boa alimentação e exercícios físicos.

PALAVRAS-CHAVE: lipólise, adiposidade, estimulação elétrica, gordura e fisioterapia.

\section{REFERÊNCIAS:}

AROCA, Graciele Guimarães Pitelli et al. Avaliação termográfica e antropométrica da eletrolipólise na adiposidade localizada. Fisioter. Mov., v. 30, n. 1, p. 29-38, mar., 2017.

LEITE, Beatriz Cordovil et al. Impacto da criolipólise associada à radiofrequência na adiposidade localizada. Fisioterapia Brasil,v.18,n.5,p.616-623,maio,2017.

MACEDO, Ana Carolina Brandt et al. Efeitos da aplicação da corrente polarizada e da iontoforese na gordura localizada em mulheres. Fisioter. Mov., v. 26, n. 3, p. 657-664, set., 2013. 


\section{EFEITOS DO MÉTODO PILATES SOBRE O EQUILÍBRIO DE IDOSAS:}

\section{UMA REVISÃO BIBLIOGRÁFICA}

Monaliza de Sousa Moura ${ }^{1}$, Mayara Monteiro Andrade ${ }^{2}$, Maria Marta Ferreira Oliveira de Sousa ${ }^{3}$, Eva Karoline Rodrigues da Silva ${ }^{4}$,Mayra Monteiro Andrade ${ }^{5}$, Wellington dos Santos Alves ${ }^{6}$

1,2,3,4 Graduando, Universidade Estadual do Piauí, Teresina, PI.

5 Técnico, Politécnico, Teresina, PI.

${ }^{6}$ Doutor, Universidade Estadual do Piauí,Teresina, PI.

E-mail do apresentador: monalizasousamoura@hotmail.com

INTRODUÇÃO: Segundo o Relatório de Desenvolvimento da Organização das Nações Unidas de 2017, o número de pessoas idosas cresce continuamente em todo o mundo. Considerando esta afirmativa e entendendo que nesse processo natural o indivíduo passa por alterações morfológicas, funcionais e bioquímicas (SILVA et al., 2017), as quedas tornam-se cada vez mais frequentes, sendo o gênero mais afetado, o feminino (ORGANIZAÇÃO MUNDIAL DE SAÚDE, 2010). Por esse motivo, a atividade física é comumente procurada por esse público, para auxiliar na manutenção da independência funcional. Entre essas atividades está o pilates, que fortalece os músculos, estabiliza as costas e músculos pélvicos, mantém a coluna alinhada adequadamente e assim, auxilia o centro de gravidade (MESQUITA et al, 2015). OBJETIVO: Verificar a eficácia do método pilates no equilíbrio de idosas. MATERIAIS E MÉTODOS: Trata-se de uma revisão bibliográfica, onde foram realizadas pesquisas no mês de novembro de 2018, mediante consulta a artigos científicos nas bases de dados eletrônicas SCIELO e Biblioteca Virtual em Saúde (BVS), com os descritores em língua portuguesa: idoso (a), pilates e equilíbrio, bem como seus respectivos termos em inglês. Foram incluídos: artigos em língua portuguesa e inglesa, entre os anos de 2008 a 2018, que investigaram acerca da utilização do pilates no equilíbrio de idosas. Foram excluídos estudos incompletos, duplicados nas bases, artigos que incluíssem outras faixas etárias sem especificá-las nos resultados (ou trouxessem idosos de forma geral sem especificar sexo), e revisões. RESULTADOS E DISCUSSÃO: Dos 52 artigos encontrados,seis constituíram esse estudo por adequarem-se aos critérios estabelecidos. CAMARGO et al (2016), avaliou os resultados do Pilates na postura estática e no equilíbrio dinâmico de idosas. Todavia, não encontrou mudanças nas variáveis analisadas antes e depois da intervenção. MARTINEZ et al (2018), comparou o equilíbrio corporal, a mobilidade e a força muscular respiratória de idosas praticantes de pilates com idosas ativas e concluiu que a avaliação do equilíbrio corporal não mostrou diferenças significativas entre as praticantes, porém, as duas atividades demonstraram resultados satisfatórios. MESQUITA et al (2015) investigou o efeito do Método Pilates e da Facilitação Neuromuscular Proprioceptiva sobre o equilíbrio estático e dinâmico de idosas e constatou melhora significativa do equilíbrio estático e dinâmico. Entretanto, não houve grande diferença entre os grupos, demonstrando que ambos os métodos podem ser utilizados. NAVEGA et al (2016), avaliou o equilíbrio de idosas praticantes do método e constatou que o grupo experimental apresentou manutenção do equilíbrio. SIQUEIRA 
et al. (2010) avaliou os efeitos que o Pilates pode oferecer ao equilíbrio estático de idosas saudáveis e obteve respostas positivas quanto à melhora desse após intervenção.SOUZA et al (2013) avaliou os efeitos de um programa de exercícios do método Pilates sobre o equilíbrio de mulheres pós-menopausa, praticantes de Pilates, e constatou um ganho de $6,9 \%$ sobre o equilíbrio nesse grupo. CONSIDERAÇÕES FINAIS: De acordo com a maioria dos achados deste trabalho, conclui-se que o pilates pode auxiliar no ganho e manutenção do equilíbrio de mulheres idosas; porém, são necessários mais estudos acerca do tema, pois ainda existem divergências entre os resultados obtidos nos artigos.

PALAVRAS-CHAVES: Idosas, pilates, equilíbrio.

\section{REFERÊNCIAS:}

CAMARGO, M.B. et al. Efeito do Método Pilates na postura e no equilíbrio dinâmico de idosas. Fisioter Bras. v.17,n.3,p.236-43.2016.

MARTINEZ,J.A.R. et al. Body balance, mobility and respiratory muscle strength in elderly women practitioners of the method pilates. J Phys Res.v.8,n.1,p.8-15.2018.

SILVA, Igor Almeida et al. Efeito de um protocolo de Facilitação Neuromuscular Proprioceptiva (FNP) no equilíbrio postural de idosas. Fisioter. Pesqui., São Paulo , v. 24, n. 1, p. 62-67, Mar. 2017. 


\title{
IMPACTOS DA ENDOMETRIOSE NA QUALIDADE DE VIDA DA MULHER
}

\author{
Sarah Lays Campos da Silva ${ }^{1}$; Abimael de Carvalho 2; Vivia Rhavena Pimentel Costa33 \\ ${ }^{1}$ Graduanda de Fisioterapia pela Universidade Estadual do Piauí - UESPI, Teresina, PI; \\ ${ }^{2}$ Graduando de Fisioterapia pela Universidade Estadual do Piauí - UESPI, Teresina, PI; \\ ${ }^{3}$ Graduanda de Fisioterapia pela Universidade Estadual do Piauí - UESPI, Teresina, PI.
}

\section{E-mail do apresentador: sarahlayscampos1@gmail.com}

INTRODUÇÃO: A endometriose é uma doença ginecológica crônica, de caráter progressivo que acomete cerca de 70 milhões de mulheres mundialmente, sendo uma das principais causas de hospitalização em países industrializados (MOREIRA \& BENTO, 2018). Nesse enfoque, no Brasil, entre 2009 e 2013, foram registradas 71.818 internações em sua decorrência (FILHO et al, 2014). Seus principais sintomas clínicos, entre eles a dor pélvica crônica, podem gerar impacto na qualidade de vida das mulheres, afetando na realização de AVDS, nas relações sexuais, no trabalho, na prática de exercícios físicos e demais atividades sociais (ALBERICO, 2013). OBJETIVOS: Analisar o impacto da endometriose na vida da mulher. MATERIAIS E MÉTODOS: Revisão bibliográfica descritiva pesquisada em periódicos nacionais e internacionais disponíveis nas bases de dados SCIELO, LILACS, BIREME e no banco de dados TRIP DATABASE no período de janeiro a fevereiro de 2019. Os descritores foram: endometriosis, women's Health, Quality of life, sendo encontrados 190 artigos nos idiomas inglês, português e espanhol. Foram incluídos: estudos de coorte, estudos de caso e controle, revisões sistemáticas, relatos de caso e ensaios clínicos controlados publicados nos últimos dez anos. Foram excluídos: revisões bibliográficas, artigos com mais de 10 anos de publicação e aqueles que não condiziam com os objetivos da pesquisa. Após a filtragem restaram 46 artigos que foram submetidos aos critérios de elegibilidade adotados para o estudo. Após a análise, 22 mostraram-se potencialmente relevantes para a composição do estudo e 2011 como maior ano de publicação. RESULTADOS E DISCUSSÃO: a endometriose se encontra associada a uma variedade de sintomas sendo os mais destacados: dismenorreia, dispaurenia, dor pélvica crônica e irregularidades menstruais. Esses sintomas possuem grande impacto no bem-estar emocional, social e físico durante os anos de vida reprodutiva da mulher. Verificou-se que a dor pélvica crônica é o fator clínico mais frequente na endometriose, sendo uma das principais preocupações das mulheres, pois é a maior responsável pelo afastamento do convívio social, do trabalho, do relacionamento com o companheiro, com a família e amigos, além de as afastarem do impulso de viver, as levando ao total desespero e aproximando-as da intenção de interrupção da vida. Outro problema é a infertilidade; $90 \%$ das mulheres acometidas possuem dificuldade para engravidar, resultando em sentimentos de inadequação, incertezas e depressão, contribuindo para o rompimento de relacionamentos. Porém, destaca-se que o apoio dos familiares, dos amigos, assim como um atendimento de qualidade nos serviços de saúde, constitui-se como fatores importantes que contribuem para a melhora da qualidade de vida das mulheres que convivem com a endometriose. CONSIDERAÇÕES FINAIS: A endometriose afeta todos os aspectos da vida da mulher ao interferir em seus relacionamentos, na sua capacidade reprodutiva e na realização de atividades habituais impactando negativamente na qualidade de vida da mesma, independentemente de sua faixa etária. Portanto, sugere-se que os profissionais de Saúde, criem espaços de ajuda mútua possibilitando a participação das mulheres, o debate e até mesmo o compartilhamento de questões sobre a endometriose e da vida com 
endometriose, buscando sanar suas dúvidas, conhecer formas de tratamento, e buscar administrar seus conflitos.

PALAVRAS-CHAVES: Endometriosis, Women's Health e Quality Of Life.

\section{REFERÊNCIAS:}

MOREIRA N. C. M; BENTO P. A S. S. Quando os olhos não veem o que as mulheres sentem: a dor na narrativa de mulheres com endometriose. Physis: Revista de Saúde Coletiva, Rio de Janeiro, v. 28(3), e280309, 2018.

MISSMER S. A.; LAUFER M. R.; SARDA V.; VITONNIS A. F.; DiVASTA A. D; GALLAGHER J. S.; The Impact of endometriosis on quality of life Adolesccents. J.S. Gallagher et al. / Journal of Adolescent Health 63 (2018) 766772, 2018. 


\title{
PERSPECTIVAS DA ATUAÇÃO FISIOTERAÊUTICA NO TRATAMENTO DO LINFEDEMA ASSOCIADO AO CÂNCER DE MAMA
}

\author{
1 Thele Albuquerque da Silva; ${ }^{2}$ Francisca Clara Lopes Soares; ${ }^{3}$ Marcelino Martins
}

\begin{abstract}
${ }^{1}$ Graduanda de Fisioterapia pela Universidade Estadual do Piauí - UESPI, Teresina, PI; ${ }^{2}$ Graduando de Fisioterapia pela Universidade Estadual do Piauí - UESPI, Teresina, PI; ${ }^{3}$ Fisioterapeuta, Doutor em Engenharia Biomédica , Universidade Estadual do Piauí UESPI, Teresina, PI.
\end{abstract}

E-mail do apresentador: thelle.albuquerque@hotmail.com

INTRODUÇÃO: O câncer de mama é o mais frequente e comum tumor maligno entre as mulheres, em termos globais. Devido ao diagnóstico precoce e modalidades de tratamento significativamente melhores nos últimos anos taxas de sobrevivência estão crescendo, resultando em um aumento na taxa de prevalência de Linfedema associado ao Câncer de Mama. A atuação fisioterapêutica tem se mostrado promissora ao longo dos últimos anos no tratamento do linfedema, atuando sobre o aumento do fluxo linfático e contribuindo para a melhora geral na qualidade de vida dessas pacientes. OBJETIVO: Identificar na literatura a atuação fisioterapêutica no tratamento do linfedema associado ao câncer de mama, baseado em publicações mais atuais. MÉTODOS: Trata-se de uma revisão sistemática realizada a partir de consulta às bases de dados eletrônicas PEDro e PUBMED utilizando os descritores câncer de mama, linfedema e fisioterapia. Foram incluídos ensaios clínicos randomizados, na língua portuguesa inglesa ou espanhola, com data de publicação a partir de 2015 com o intuito de analisar quais o que tem sido ultimamente estudado com relação ao tema. A pesquisa resultou em 223 ensaios clínicos, após análise minuciosa por dois avaliadores independentes e cegos, 19 foram selecionados atendendo aos critérios de inclusão e elegibilidade guiados por uma ficha padronizada. As discordâncias foram examinadas por um terceiro revisor. A qualidade metodológica dos artigos foi avaliada através da escala PEDro. RESULTADOS: Identificou-se que os estudos encontrados focam em três perspectivas principais: a primeira relacionada a comparação entre técnicas da fisioterapia (10 artigos); a segunda, relacionado ao estudo de métodos isolados (4 estudos), e por fim os que buscam adicionar uma outra intervenção à Terapia Linfática Descongestiva Completa (tida como terapia padrão) e então determinar se há efeitos adicionais, que somam 5 estudos. Os principais resultados positivos para a redução do grau de linfedema foram com Drenagem Linfática, exercícios ativos, principalmente aquáticos, mangas de compressão, acupuntura quente e Terapia Extracorpórea de Ondas de Choques. Quando ao efeito adicional ao tratamento padrão as únicas intervenções que se mostraram úteis foram Exercícios Clínicos de Pilates e Laser de Baixa Intensidade. Também foram encontrados resultados positivos para aumento da Amplitude de Movimento, força muscular e, consequentemente, qualidade de vida. CONSIDERAÇÕES FINAIS: Os resultados, portanto, demonstram que a atuação fisioterapêutica no tratamento do linfedema é ampla e a literatura atual volta suas perspectivas para o interesse de potencializar os resultados das intervenções já estabelecidas de modo a proporcionar a melhora da capacidade funcional e mobilidade e garantir maior qualidade de vida para 0 paciente. 
PALAVRAS-CHAVE: câncer de mama, linfedema e fisioterapia.

\section{REFERÊNCIAS:}

ABDELHALIM, N. M. et al. Comparison of Extracorporeal Shock Waves Therapy Versus Intermittent Pneumatic Compression Therapy in Breast Cancer Lymphedema. Int. Journal of Cancer Research.14(2);77-85 2018.

DE VRIEZE, T. et al. Protocol of a randomised controlled trial regarding the effectiveness of fluoroscopy-guided manual lymph drainage for the treatment of breast cancer-related lymphoedema . Europ. Jour. of Obstetrics \& Gynecology and Reproductive Biology; 12(2), 2017.

OCHALEK, K. et al. Do Compression Sleeves Reduce the Incidence of Arm Lymphedema and Improve Quality of Life? Two-Year Results from a Prospective Randomized. Lymp. Res. and Biology. 49(2), 2017. 


\title{
PESQUISA ETNOFARMACOLOGICA SOBRE O USO DE PLANTAS MEDICINAIS E FITOTERÁPICOS POR MULHERES NO AUTOCUIDADO
}

\author{
Alice Bispo dos Santos ${ }^{1}$; Hildelanne Soares de Barros ${ }^{1}$; Ruy Gabriel Costa \\ Sousa'; Alessandra Camillo da Silveira Castello Branco² \\ ${ }^{1}$ Centro Universitário Santo Agostinho, Teresina, Piauí; \\ ${ }^{2}$ Doutora em Produtos Naturais e Sintéticos Bioativos, Centro Universitário Santo
} Agostinho, Teresina, Piauí;

E-mail do apresentador: hildelanne@gmail.com

INTRODUÇÃO:O uso das plantas medicinais para prevenção, tratamento e cura de enfermidades tem crescido muito nos últimos anos. De acordo com a literatura, esta tradição é perpetuada, sobretudo, pelas mulheres da família, principalmente as idosas, visto que assumem tradicionalmente a responsabilidade pelos cuidados no contexto familiar. OBJETIVOS:Avaliar o uso de plantas medicinais e medicamentos fitoterápicos por mulheres moradoras do Bairro São Pedro no município de Teresina - Piauí. MATERIAIS E MÉTODOS:O presente estudo, aprovado pelo o comitê de ética e pesquisa em humanos com o número 82821618.2.0000.5602, se trata de uma pesquisa do tipo transversal e descritiva com uma abordagem quantitativa e qualitativa. Foi realizado com mulheres moradoras do Bairro São Pedro do município de Teresina do estado do Piauí, com a utilização de um questionário como instrumento de pesquisa aplicado por domicilio em quadras sorteadas. Buscou-se envolver mulheres de todas as faixas etárias que faça uso de plantas medicinais e medicamentos fitoterápicos. RESULTADOS E DISCUSSÃO: Foram entrevistadas 54 mulheres de idade média de 47 anos,corroborando com os estudos analisados, e com um média de $\geq 5$ moradores por casa e renda familiar entre 2-3 salários mínimos, fatores estes que influenciam no uso das plantas medicinais, considerado que elas possuem baixo custo e são de fácil acesso.Dentre as entrevistadas, $79,6 \%$ relataram fazer ou já ter feito o uso de plantas medicinais e que veio de hábitos familiares $(92,6 \%)$, isto se deve pôr a família ser pioneira na transmissão de conhecimento e cuidados sobre a saúde e da difusão da tradição de cultivo de plantas medicinais com o intuito de facilitar a obtenção de recursos terapêuticos. Foram relatadas 54 plantas, cultivadas em sua maioria no próprio quintal ou no de vizinhos, sendo a erva cidreira, boldo, camomila e hortelãos mais utilizados respectivamente, estes resultados foram similares aos encontrados em literatura, porém em quantidades e ordem diferente podendo estar relacionado contradições inerentes a cada região. O modo de preparada mais empregado foi por decocção e infusão, sendo a folha a parte mais utilizada, hipoteticamente devida a sua constante disponibilidade, todavia o uso de decocção para esta parte da planta não é indicado, pois pode inativar a sua atividade terapêutica. Das entrevistadas, $68,5 \%$ relataram possuir problemas crônicos de saúde como Hipertensão $(35,1 \%)$ e Diabetes $(21,6 \%)$, no entanto o uso das plantas em sua maioria foi para fins alimentícios e tratamento de condições autolimitadas que, no que se refere a indicação, algumas estavam adequadas, parcialmente adequadas e inadequadas. Em relação aos medicamentos fitoterápicos nenhumas das entrevistadas relataram uso. CONSIDERAÇÕES FINAIS: Dado o exposto, foi possível observar que o uso das plantas medicinais por estas mulheres, em alguns casos, estava em desacordo com a literatura, demonstrando que a informação cientifica sobre determinadas plantas ainda se mostra de forma escassa na população e que há a necessidade de uma maior orientação por parte do serviço público de saúde. 
PALAVRAS-CHAVES: Mulheres, Plantas Medicinais, Medicamentos Fitoterápicos.

\section{REFERÊNCIAS:}

SCHIAVO, M. et al. Conhecimento sobre plantas medicinais por mulheres em processo de envelhecimento. Semina: Ciências Biológicas e da Saúde, v. 38, n. 1, p. 45-60, 2017.

SCHWAMBACH $\mathrm{K}, \mathrm{H}$. Utilização de plantas medicinais e medicamentos no autocuidado no município de Teutônia, RS. 2007. 98p. Dissertação (Mestrado em Ciências Farmacêuticas) - Universidade Federal do Rio Grande do Sul, Porto Alegre, 2007.

SOUZA, J. S. S. et al. Uso de plantas medicinais por comunidades do município de curitiba. Divers@!, v. 10, n. 2, p.91-97, 2018. 


\title{
PERFIL EPIDEMIOLÓGICO DE CÂNCER DE COLO DE ÚTERO NO ESTADO DO PIAUÍ, NOS ANOS DE 2012 A 2016
}

\author{
Luís Eduardo de França Barros Menezes ${ }^{1}$; Manuela Soares Vasconcelos ${ }^{1}$; Eliamara \\ Barroso Sabino ${ }^{2}$
}

1 Centro Universitário UNINOVAFAPI, Teresina, Piauí;

2 Doutora em Biotecnologia, Centro Universitário UNINOVAFAPI, Teresina, Piauí;

E-mail do apresentador: luiseduardobarros@outlook.com

INTRODUÇÃO: O câncer do colo do útero ou cervical é um tumor maligno encontrado na parte inferior do útero. Tal câncer é causado pela infecção persistente oriunda de alguns tipos do Papiloma Vírus-HPV, sendo esses, chamados de tipos oncogênicos $(6,11,16$ e 18) . Portanto, é de suma importância traçar o estudo epidemiológico desta neoplasia maligna que é a terceira mais comum entre mulheres. OBJETIVO: O objetivo deste trabalho é analisar a elevada incidência de casos de câncer de colo de útero entre os anos de 2010 e 2016 no estado do Piauí. É indubitável, também, ressaltar a importância da prevenção, já que a cura está relacionada ao momento precoce do diagnóstico e ao estadiamento da doença. MATERIAIS E MÉTODOS: Para a confecção deste trabalho, do tipo quantitativo descritivo e de razão epidemiológica, foram utilizados dados do sistema de informações do SUS (DATASUS), na subseção do Sistema de Informação do Colo de Útero (SISCOLO). O período referente aos dados coletados foi do ano de 2010 a 2016, restringindo-se aos casos de câncer colo-uterinos no estado do Piauí, sem restrição à idade das pacientes. Os resultados foram traçados em tabelas, de acordo com cada ano analisado. RESULTADOS: Foram registrados, entre 2010 e 2016, 869 mortes de mulheres no estado do Piauí vítimas de câncer de colo de útero, levando em consideração a localização primária do tumor. A faixa etária com mais óbitos foi a de 50 a 59 anos, com 200 casos, seguido da faixa de 60 a 69 anos, com 164 casos. Por sua vez, a faixa etária com menor valor de casos foi a de 15 a 19 anos, com apenas um caso registrado no estado. As taxas por 100 mil habitantes chegou a $10,38 \%$, quase suas vezes maior que a taxa nacional que foi de $5,92 \%$, no mesmo período. Constata-se, assim, que o estado do Piauí, apesar de não possuir os maiores números de casos do país, é um dos que apresenta a maior taxa de mortalidade frente à taxa nacional e aos demais estados da nação. CONSIDERAÇÕES FINAIS: Em vista dos dados apresentados e da constatação da alta prevalência de casos de câncer de colo de útero em mulheres piauienses, faz-se necessária a ampliação de medidas de prevenção a esta patologia que, conforme apresentado, apresenta alta letalidade em caso de diagnóstico confirmado em estágio avançado, e alto percentual de cura, se diagnosticado com antecedência. Colabora-se, assim, com o incentivo às políticas públicas governamentais para ampliarem as campanhas, a exemplo do Outubro Rosa, e da facilitação do acesso das mulheres para a realização de exames citológicos preventivos a serem realizados na Atenção Primária à Saúde.

PALAVRAS-CHAVES: Colo de útero; mortalidade; câncer

\section{REFERÊNCIAS:}

INSTITUTO NACIONAL DE CÂNCER (Brasil). Estimativa 2018. Incidência do Câncer no Brasil. Rio de Janeiro: INCA, 2017. Disponível em: 
http://www.inca.gov.br/estimativa/2018/estimativa-2018.pdf Acesso em:21/01/2019. INSTITUTO NACIONAL DE CÂNCER (Brasil). Atlas da Mortalidade. Disponível em: https://mortalidade.inca.gov.br/MortalidadeWeb/. Acesso em: 21/01/2019. 


\title{
IMPLICAÇÕES DA EPISIOTOMIA EM MULHERES PRIMÍPARAS
}

\author{
LOPES, Caroline Sousa ${ }^{1}$; SOUSA, Dalila Marielly Alves ${ }^{2}$; CARCARA, Lorena Rocha \\ de Abrantes 3 ; AQUINO, Maria Luiza da Silva ${ }^{4}$; REIS, Mayra Dayananda Cunha ${ }^{5}$; \\ ROCHA, Silvana Santiago da ${ }^{6}$ \\ 1 Universidade Federal do Piauí-UFPI, Teresina, Piauí; \\ 2 Universidade Federal do Piauí-UFPI, Teresina, Piauí; \\ 3 Universidade Federal do Piauí-UFPI, Teresina, Piauí; \\ 4 Universidade Federal do Piauí-UFPI, Teresina, Piauí; \\ 5 Universidade Federal do Piauí-UFPI, Teresina, Piauí; \\ ${ }^{6}$ Pós-doutorado em Enfermagem, Universidade Federal do Piauí-UFPI, Teresina, \\ Piauí;
}

E-mail do apresentador: maria_luiza200@live.com

INTRODUÇÃO:A episiotomia é um procedimento realizado desde o século XVIII com o intuito de auxiliar em casos de dificuldades nos partos, apenas em situações necessárias. No entanto, nos dias atuais a episiotomia vem sendo realizada como um procedimento de rotina, mesmo havendo estudos que apontam essa prática como ineficaz e danosa. No caso de mulheres primíparas esse procedimento se torna ainda mais delicado tendo em vista que é o seu primeiro parto, a falta de informação e instrução dos profissionais da saúde para com a parturiente -que ocorre frequentemente- pode resultar em uma experiência traumática. Pois a submissão à uma episiotomia sem necessidade pode trazer além de danos físicos, danos psicológicos, fazendo- se necessário a transparência e clareza para com a parturiente. OBJETIVOS: Identificar através das produções literárias as implicações da realização da episiotomia em mulheres primíparas. MATERIAIS E MÉTODOS: Revisão integrativa da literatura, realizada em dezembro e janeiro de 2018 e 2019. Para seleção dos artigos utilizou-se bases de dados de literatura Latino-Americano e de Caribe em ciências da saúde (LILACS); Scientific Eletronic Library Online (SCIELO) e Medical LiteratureAnalysisandRetrieval System Online (MEDLINE). Foram critérios de inclusão artigos em inglês e português, com textos completos, nos anos de 2015 a 2018, com os descritores: episiotomia; transtornos puerperais; lacerações; complicações do trabalho de parto. Foram encontrados 128 dos quais 24 atenderam aos critérios de inclusão que constituem a amostra final. RESULTADOS E DISCUSSÃO: A episiotomia vêm sendo realizada em grande número de partos vaginais com o objetivo de aumentar a abertura vaginal e prevenir lacerações na vagina e períneo. Mas observa-se que esse procedimento tem sido realizado predominantemente em primíparas tendo como implicações, além do trauma e da dor perineal, a não prática de relação sexual devido ao medo da aparência de sua vagina bem como o aumento das chances de episiotomia e lesões em parto posterior. CONSIDERAÇÕES FINAIS: Conclui-se que taxa de episiotomia encontra-se acima das recomendações da OMS e que não deve ser uma prática rotineira, uma vez que é desencadeadora de diversas complicações seja na saúde, sexualidade ou autoestima da mulher.

PALAVRAS-CHAVES: Episiotomia; Transtornos puerperais; Lacerações; Complicações do trabalho de parto. 


\section{REFERÊNCIAS:}

BALLESTEROS-MESEGUER, Carmen et al . Episiotomia e sua relação com diferentes variáveis cínicas que influenciam sua realização. Rev. Latino-Am. Enfermagem, Ribeirão Preto , v. 24, e2793, 2016.

MACÊDO, Lorena Carneiro de. Avaliação da função sexual em primíparas após parto vaginal e nuligestas. Revista Pesquisa em Fisioterapia, Bahia, no 7, p. 24-29, 2017.

PEÑA, Solange Ribeiro. GOMES, Célia Regina de Godoy. Episiotomia e suas implicações. Arquivos do MUDI, Paraná, v. 20, № 1, p. 25-37, 2016. 